\title{
Learning from experts: Fostering extended thinking in the early phases of the design process
}

\author{
Grietjie Haupt \\ Department of Science, Mathematics and Technology Education, University of Pretoria, Hillcrest 2000, South \\ Africa \\ e-mail: grietjie.haupt@up.ac.za
}

\begin{abstract}
Connecting the inner processes with outer information
Empirical evidence on the way in which expert designers from different domains cognitively connect their internal processes with external resources is presented in the context of an extended cognition model. The article focuses briefly on the main trends in the extended design cognition theory and in particular on recent trends in information processing and embodiment theory. The aim of the paper is to reflect on the implications of an understanding of expert design cognition as an extended system, which can account for complexity and nonlinearity in design thinking and problem-solving, for technology and design education. This is achieved by showing the relevance of the cross-correlations and the dynamics involved at the intersection of cognitive phases, intention-driven decision making and embodiment principles of experts for novice education in technology and design. It is argued that $20^{\text {th }}$-century onesided approaches to design education no longer adequately serve the needs of $21^{\text {st }}$ century. It is further argued that a combined information-processing+embodiment approach may be the answer. The article presents salient results of a case study using think-aloud-protocol studies in a quasi-experimental format that was used as it has proven to be a central instrument yielding scientific data in the cognitive science paradigm. Results suggested extended design environments may be particularly well-suited to the mediation of design thinking. Finally, based on these results, the article examines how educators can exploit the combined approach to advance the making of connections between the inner and outer world in design education.
\end{abstract}

\section{Keywords}

Design cognition, expert, extended cognition, early phases, information processes, embodiment.

\section{Introduction}

This study involves the proposal of an alternative combined conceptual framework for studying design protocols of experts that may assist in devising alternative ways of reflecting on design education strategies. The framework was successfully applied in a PhD project (Haupt 2013) from which this study draws. The central assumption of this framework is that design cognition extends over centralized information processing systems and encompasses sources of external information including people, artifacts and natural objects in the environment. The framework allows for real-world complexity and ill-structuredness of design problems, which is described further on in more detail. In particular I support Spiro, Feltovich, Jacobson and Coulson (2013) argument that:

- The complexity and design task-to-task irregularity or ill-structured nature of certain types of problems poses serious problems for traditional one-sided approaches to instruction.

- Cognitive and instructional disregard of problems related to complexity and variability in patterns of information access and use leads to learning failures that 
develop into common, predictable forms. These forms are distinguished by conceptual oversimplification and the inability to transfer knowledge to new problems.

- The resolution for such insufficiency requires the fostering of learning processes that allow for greater cognitive flexibility.

The genesis of this article rests in a unique set of data collected by the author during the conduct of a $\mathrm{PhD}$ study in which the cognitive dynamics of three small groups of expert designers from architecture, industrial designing and mechanical engineering applying a mixed methods approach. The case study entailed two to three hours of think-aloud protocols involved the production of verbal utterances and sketches during the early phases of the design process, namely problem structuring and problem solving. The author used an alternative conceptual framework in which the information processing (Goel \& Pirolli, 1992) and embodiment principles (Richardson, Shockley, Fajen, Riley, \& Turvey, 2008) were combined to investigate the way in which expert designers synergistically move between their internal and external sources of information. The purpose of this article is to reflect on the usability of such an alternative model of cognition by discussing its use in experts' design process. The long term aim of such an alternative model is to assist in eliminating possible conflicting dualist approaches to design cognition in design education. It might thus contribute to a better insight in potential instructional strategies that may be more conducive to fostering adaptability, flexibility and effective access and use of a variety of information sources during the early phases of students' design projects. My position in this paper is that designers, irrespective of domain or expertise, need to develop mental flexibility (Spiro et al., 2013) yet coherence (Kearsley, 1998) in their thinking, in order to move adaptively between internal processes and external information when structuring and solving design problems. The assumption is furthermore that much can be learned from experts to inform our design education programmes (Cross, 2004; de Vries, 2005).

\section{Brief overview of key concepts}

Some key concepts are contextualised in order to understand the theoretical underpinnings of this article.

\section{Expertise}

Various authors, from different perspectives (Lawson \& Dorst, 2009; Popovic, 2004; Simonton, 2003), have attempted to define the concept 'expertise'. In a design context, researchers including Cross (2004), Christiaans and Venselaar (2005) and Lawson and Dorst (Lawson \& Dorst, 2009) contributed valuable insights. In this paper, I consider the following key principles of design experts' knowledge and problem solving abilities, adapted from Bransford, Brown \& Cocking (2000) relevant:

- Experts notice features and meaningful patterns of information in their extended environments, including their external representations, which are not noticed by novices.

- Experts have acquired a great deal of content knowledge that is organised in ways that reflect a deep understanding of their subject matter.

- Experts' knowledge cannot be reduced to sets of isolated facts or propositions but, instead, reflects contexts of applicability through recognition of determinant interrelationships between information. 
- Experts are able to flexibly and with little conscious effort retrieve important aspects of their knowledge that coherently relate to their intentions.

These abilities all allude to complex thinking skills and imply many hours of practice. However, we do not seem to understand yet how that is acquired by novices, in a manner that reflect higher order thinking. From current technology education literature it is evident that there is a gap in the research, which can, according to Petrina (2007), be attributed to the primary interest of education researchers in students' general acquisition of technological literacy, instead of on their higher order cognitive activities. Authors such as Natarajan (2007) claims that such a superficial research agenda results in confusion about what is essential about 'learning to design', when reporting on best practices. In addition, it also leads to misconceptions and incorrect priorities in curriculum design (Petrina, 2007; Stables, 1997). It therefore seems important to understand the primary underpinnings of higher order complex thinking.

\section{Design cognition theory}

Conflicting definitions and descriptions of the activity of 'design' exist in literature about design research as well as how curriculum documents and textbooks in design education, leading to a confusing picture of the cognitive aspects thereof. This seems to be the result of modeling designing as linear, as opposed to multidirectional and iterative (Kimbel, Stables \& Green (1996). In order to avoid confusion, I follow the Terzidis' (2007) explanation of the etymological origins of the verb 'design'. Its roots are to be found in the Latin prefix 'de' that means 'derivation, deduction or inference' combined with the Latin word 'signare', namely 'conceptualisation, imagination and interpretation'. When combined with the Greek word $\sigma \chi \varepsilon \delta 10$ (pronounced schedio) for 'design', which is seen as the approximate capturing, conceiving, and of an expectation (Terzidis, 2007: 69), it leads to an understanding that 'designing' on all levels of expertise implies having an intention (Anderson, 2003; Simon, 1969) to outline the main features of a potential solution to a problem in a particular context by designing useful physical objects or artifacts that might change existing situations. At the centre of this understanding is the paradoxical simultaneous notions of 'incompleteness, vagueness or ambiguity, yet also about likelihood, expectation. As such, the notion of intention is key (Simon, 1996). Designing as a problem solving activity is typically nonroutine, irregular or ill-structured. Therefore designing is not trivial (Visser, 2004) and is not the same as solving mathematics problems, construction problems, or visualisation problems and requires its own unique kinds of cognitive processes (Blessing \& Chakrabarti, 2009). This means that educators should emphasise and capitalize on its uniqueness and attempt to understand the psychological influence it has on the design process and the subsequent representations of students (Goel, 1995). Ignorance of complexity is neither conducive to student work nor representative of how design thinking in real life works. One way of addressing this is to consider designing as a process of extended cognition, a metaphysical concept in the psychology literature of problem solving as used by authors such as Shani (2012) and Marsh and Drayson (2010). I thus propose a productive model as conceptual framework dealing with this challenge by combining two seemingly conflicting approaches to cognition (Kirsh, 2009), namely information processing and embodiment, which I discuss in the following section. In order to understand the cognitive complexity of these processes, it is necessary to clarify the two primary approaches to designing, namely information processing and ecological interaction. 


\section{Extended cognition and its historical roots}

Extended cognition assumes that solving design problems are explained in terms of an integration of traditional information processing theory with ecological theories advocating embodiment as a source of external information. The implication is that internal processes take place in a mental problem solving space where a particular task environment that extends over internal (mental) and external situational) environments drives the problem solving process. I adopted this theory as point of departure for this article.

Information processing theories as they relate to problem solving were established by Simon (1969) in the late 1960's. These theories are broadly based on the assumption that 'thinking' is solely governed by the brain and subsequent internal processing of information. The consequence is that the 'mind' and the 'body' are seen as two separate entities. It furthermore views 'thinking' as something taking place in a rule governed symbol system in which humans use representations to show what they think and how they understand the world around them (Goel, 1995). This implies that human behaviour is dependent on internal processes based on rational, intentional, systematic and linear planning (Miller, Galanter, \& Pribram, 1960). The implication is further that a plan is considered as a list of instructions that can control the order in which a sequence of operations is to be performed, implying linearity and sequentiality. However, there is general consensus amongst design researchers (Blessing \& Chakrabarti, 2009; Gero, 1996; Schön, 1987) that this is not how authentic design work takes place, and therefore begs an alternative modeling thereof.

\section{Control structure}

Together, the constructs 'control structures', 'personal stopping rules' and 'evaluation functions' are essential psychological characteristics of the early phases of the design process. The psychological understanding of expert designers' control structures is that it is relatively loose, allowing them to consider multiple contexts and possible ideas without fixating on one idea early in their process. In addition, their loose control structure can be connected to their ability to expand their consideration of design aspects as they negotiate information from alternative sources when they structure and solve their given design tasks. The converse of 'loose' control structure is 'tight structure', which is typically found under scientific experimental conditions where instructions and other aspects of laboratory control define 'boundaries' that limit the behavioural options of participants in such experiments. However, design experiments do not comply with these conditions and as the boundaries provided in design briefs are insufficient and ill-structured, the control structures of designers are loose, giving them much freedom.

\section{Personal stopping rules and evaluation functions}

Personal stopping rules refer to subjective preferences of designers that influence the aspects and elements they focus on when deliberating alternative ideas, making design decisions and developing ideas. These subjective personalized rules are connected to designers' worldviews, personal beliefs, and preferences. As there are no right or wrong answers in designing, designers judge their decisions -in terms of appropriateness and fit-for purpose. Therefore the evaluation functions and stopping rules that they apply are typically derived from personal experience and immersion in the project.

\section{An integrated conceptual framework}

Opposing centrally governed information processing theories, embodiment theories developed from critics who approach cognition from an ecological perspective, pioneered by Gibson (Golonka \& Wilson, 2012) in the late 1970's. The essence of embodiment theories is 
that humans/designers are in a systemic relationship with the environment. As such the environment is considered as a source of information on which designers react. This implies that thinking manifests as 'doing' rather than through storing and retrieving conceptual knowledge, which in turn points to the recognition that thinking cannot be separated from its context. My own position is that to explain the complexity of designing, we need both these theories to avoid one-sided reductionist accounts thereof, as highlighted by Kirsh (2009). Conceptualising an integrated model of a combined framework to research and use as teaching guide, I combine Goel and Pirolli's (1992) information processing theory of design task environment and design problem solving space with elements of Richardson, Shockley, Fajen, Riley and Turvey (2008)'s principles of embodiment, subscribed by Anderson (2003), as presented in Figure 1.

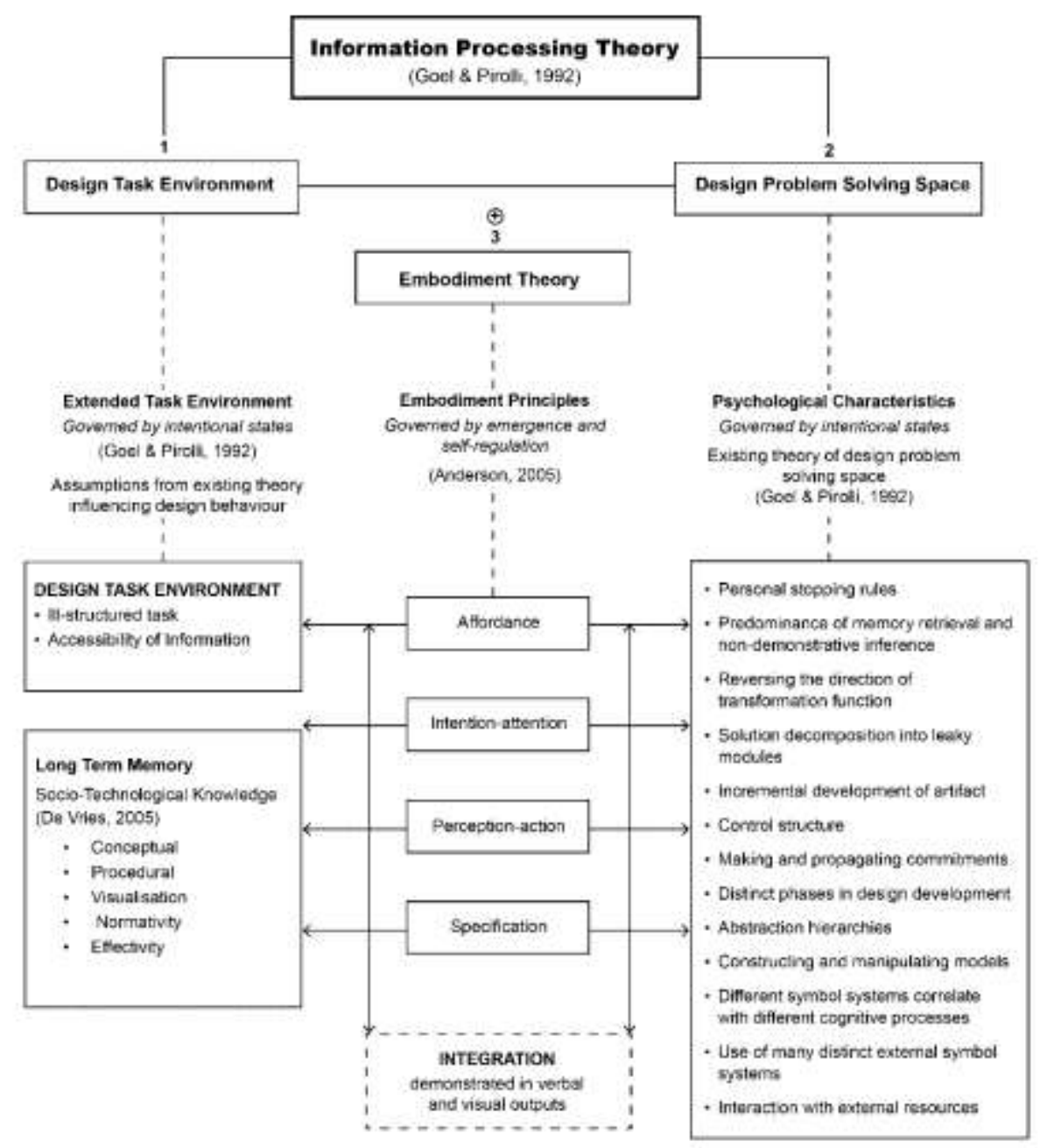

Figure 1. Conceptual framework: Extended design task environment

The framework presented here comprises three main components. Component one on the left and component two on the right, both originate from Information Processing Theory. Component one involves the idea of design task environment. However, for the purpose of this article, I integrate the conventional notion that the task environment consists of internal elements including internally stored knowledge and abilities to access such information, with 
that of externally sources of information including physical objects and the biological processes involved in the observation thereof. In a design context, the task environment encompasses complex processes of transforming a state of dissatisfaction (problem/need) to satisfaction (idea for a solution) (Simon, 1996). This is made possible by designers accessing and processing internally stored knowledge of multi-disciplinary nature (de Vries, 2006). The second component, problem solving space, represents multiple psychological characteristics empirically observed in expert design studies by Goel \& Pirolli (1992). Part three, at the centre of the figure, contains some elements of embodiment principles as Richardson et al (2008:161) defined it and were further explained by Anderson (2003:161). The elements selected for this article form the conceptual bridge between the opposing theories of Information Processing and Embodiment. The argument in this framework is that the three components are intricately intertwined and consist of complex interrelationships and hierarchical relations of determinism, which is not explained in this paper, but can be accessed in the original study (Haupt, 2013).

\section{Component One: Design task environment and embodiment}

Newell and Simon (1972) proposed the notion 'task environment' to indicate an abstract structure corresponding to a problem. Kirsh (2009, p. 266) describes it as "theoretical projection that let researchers interpret problem-solving activity in concrete situations". As such the terms 'problem' and 'task' are interchangeable (Kirsh, 2009). From an embodiment perspective 'environment' in turn refers to the notion of people's constant attempts to improve their task performance by adapting to the constraints of the environment. This means that 'design task environment' involves the entire design task given to designers, comprising the cognitive accessing of information. This encompasses recalling generic and domain specific knowledge internally stored in their long and short term memory and their ability to apply it when they structure and solve their design problems. Intertwined with this idea, is the fundamental assumption of the constructivist notion of embodiment that whenever designers encounter a particular design situation, they use a combination of experiential and situational factors to assist them in defining the problem or task (Lovett \& Anderson, 1996).

While conventional information processing theory identifies what comprises valid actions regarded as problem solving behaviour in a task environment, I agree with ecological theories that consider any action taken by a problem solver, and any external instruments used during the course of the problem solving process as potentially able to influence the process. This means that any external tools that designers use during the early phases of the design process is regarded as internal scaffolds (Goel \& Pirolli, 1989). Such action thus counts as part of the problem solving process.

Understanding design task environments requires appreciation of the nature of real world non-routine design problems. They are considered ill-structured based on the lack of information (Reitman, 1964) about the precise nature of the artifacts, or of the context or of the way in which it should be designed. This results in designers not understanding what they have to do or how they have to do it, or what the end result of their thinking and activities are expected to be, which leaves them with a general feeling of uncertainty. A relatively simplistic example, but one which is applicable in a technology education context, of such an ill-structured problem is:

'Design a water carrying device for people in rural areas'.

From this it is clear that designers do not have any information regarding who the 'people' are. They have to find out if they are women, children, elderly people or males. They further 
need to investigate whether the device needs to be self-propelled or pushed, carried or pulled by the people using it or is the choice theirs? They further will have to find out what the nature of terrain is over which the device will be carried: is it smooth, rough, dust, gravel or tarred? From this it is clear that the actual device can only be successfully designed once the contextual information has been acquired by designers, and they know what the scope of the problem is. Finding this information constitutes the problem structuring phase of the design process. Achieving this, means that designers' initial uncertainty is incrementally reduced.

\section{Component Two: Design problem solving space}

The problem solving space as framed here and applied to the early phases of the design process only, is complex and assumes that the types of problems designers are required to solve, is ill-structured, non-routine (Goel, 1995). Two cognitive phases in designers' early phases of the design process consist of problem structuring and problem solving, each characterised by its own complexities and psychological characteristics. However, gathering and processing information, according to Goel (1995), results in 'leaky cognitive instances' where designers constantly move between problem structuring and generating design solutions until the two processes converge to the commitment of a suitable solution. Many expert studies further agree that experts usually are able to distinguish between the types of information needed in these two phases.

For the conceptual framework of this paper (Figure 1), I used Goel and Pirolli's (1992) twelve invariant psychological features characteristic of experts across disciplines' problem solving space. These were adapted to constitute the following:

1. Problem structuring

2. Distinct phases in design development

3. Reversing direction of transformation function

4. Solution decomposition into leaky modules

5. Incremental development of artifact

6. Control structure and evaluation functions

7. Making and propagating commitments

8. Personal stopping rules and evaluation functions

9. Predominance of memory retrieval and nondemonstrative inferences

10. Constructing and manipulating models

11. Abstraction hierarchies

12. Use of many distinct external symbol systems (symbol systems correlate with different cognitive processes)

13. Interaction with external resources

The purpose driving this article, namely to derive educational relevance to cross-correlations and the dynamics found at the intersection of cognitive phases, intention-driven decision making and embodiment principles of expert designers (Haupt, 2013). For this reason I focused on the first two items in the list above, namely problem structuring and distinct phases in the development of solutions and its overlap with items 6 and 8, namely control structure, evaluation functions and personal stopping rules. These are explained in the following section. This focus was selected due to its direct relevance to decision making, and intentionality in design work. However, it is important to keep in mind that all twelve 
characteristics overlap and are in intricate ways interrelated to each other. These complexities are not explained in this article but can be viewed in Goel's (1995) original work and the on the expansion thereof (Haupt, 2013).

\section{Distinct cognitive phases}

Although some studies indicate that the process of problem structuring typically proceeds through many minute steps (Goel \& Pirolli, 1992), for practical reasons of this article, I only focus on the two steps involved in my empirical study. The first step involves the way in which designers access internal knowledge and use external resources to structure design problems. The second step entails the way in which designers negotiate internal and external knowledge through transformation of direction to generate and develop preliminary solutions.

\section{Problem structuring}

The first cognitive phase entails the notion of 'problem structuring', which means that designers try to understand what the problem is that they need to solve, what its scope is, and what its constraints, requirements and specifications are. Due to design problems' illstructured nature this particular cognitive phase is essential to design thinking. However, as problem structuring is not a plainly identifiable phase of the design process, but rather consisting of a series of repeated activity it is not always easy to distinguish between problem structuring and problem solving. Problem structuring in itself is not regarded as a problemsolving pursuit. However, the extent to which it is successful determines the number of subproblems designers immerse in. In addition, the extent to which problem structuring is fruitful determines how designers proceed with problem solving activities.

\section{Problem solving: preliminary design}

Equally complex is the cognitive phase of problem solving, which can be divided in three sub-phases, preliminary design, refinement and detailing (Goel \& Pirolli, 1992). In this article, I deal with preliminary design only. In this sub-phase, designers generate and explore the alternative solutions that surface during the problem structuring phase. Despite seeming misconceptions regarding creative processes (Barak \& Hacker, 2011) literature abounds that experts usually do not consider many alternative solutions. Ericsson (2006), in expert studies, ascribes this phenomenon to experts' vast experience and ability to make connections with knowledge stored in their long-term memory. The solutions that they generate are often without depth and only developed at the time of generation. Possible solutions emerge through gradual expansion and transformation of a few core ideas involving people, objects and contexts. These core ideas are usually solutions to other problems that an expert designer has encountered previously. Ideas may also stem from other life experiences suggested by associations with ideas and images brought into the problem space. Ideas may thus be out of context and even inappropriate.

Designers' flexibility can also often be observed in their iterative adaptation of their behaviour by changing the direction of transformations laterally and vertically, involving many backwards and forwards movements (Goel, 1995) thereby making copious unusual and unpredictable connections and associations with new and old information. They tend to transform their representations in order for the ideas to become useful and appropriate for a current problem (Goel, 1995). Transformations during this phase of the process are more likely to be lateral than vertical due to the exploratory nature of the phase. The duration of the incremental development process of solutions is considered a function of the information available (Newell \& Simon, 1972) to designers. This flexibility is closely connected to their loose control structures discussed further on. It is furthermore acknowledged that, as expert 
designers possess more and better developed mental resources in the form of long-term memory and working knowledge, they would complete the preliminary design phase in less time than inexperienced designers (Popovic, 2004; Simonton, 2003).

Although much of what is known about the design behaviour of designers in the early phases of the design process is derived from the computational notion that designing is a linear and sequential process consisting of two primary phases, namely problem structuring and problem solving (Simon, 1996), much evidence from empirical research indicates convincingly that in reality there is no clear division between the two early 'phases'. These phases constantly intermingle and overlap or even involve simultaneous cognitive activity, which Goel (1992) calls 'leaky modules'. This means that design problem solving is not a linear process going from analysing 'the' problem specifications to synthesising 'the' solution. Problems do not pre-exist to solutions, both are built up and elaborated simultaneously (Visser, 2004). It has further been found that, even in the event of the design brief being satisfied by a design being highly specified, design situations are always partly indeterminate (Goel, 1995). In order to structure a design problem by redefining it, designers typically frame a problematic design situation by setting its boundaries, selecting particular elements involving people, objects, contexts and their relations for attention (Vincenti, 1990). In this way designers tend to impose coherence on the problem space that guides subsequent problem solving activities (Bickhard, 2008). Research further highlights that the actual process of designing will continuously trigger awareness of new criteria for design: so-called 'problem solving triggers' (Visser, 2004) which might include the knowledge accessed by perceiving the physical environment (Ericsson \& Simon, 1993; Gibbs, 2005). Making appropriate connections between the external triggers and internalized knowledge, is in part made possible by applying loose control structures, personal stopping rules or bias and evaluation functions, when engaging in embodiment principles.

\section{Control structures, personal stopping rules and evaluation functions}

The constructs 'control structures', 'personal stopping rules' and 'evaluation functions' are closely connected and relate to commitments, or non-commitments that designers make to ideas that they generate. It stems from the broader theory of 'control conducting behaviour', which has traditionally been associated with internal processing. As such, efficient causality as metaphor to explain how mental incidents affect problem solving behaviour (Goel \& Pirolli, 1992) underpins these psychological notions. However, taking an interactive approach of perception-action cycles implies that perception plays a role in causality (Kim, Kim, Lee, \& Park, 2007). Gero and McNeill (Gero \& McNeill, 1998) have developed a model through the use of situated cognition, which describes the cycles of designers' interpretation of their environment as interconnected sensation, perception and conception processes. In order to explain these cycles in terms of parallel processes, these authors imply data and expectations as possible causes for the actions of designers. No situational studies I consulted thus far, however, connect cyclical reasoning with the loose nature of designers' control structures, which is the focus of this article. The focus of this section, thus, is not to explore selfregulating feedback loops in the perception-action as in situated studies, but rather to explain the way in which various sources of action interacted and established coherence despite the loose control structures of designers.

From a psychological perspective, a typical understanding of designers' control structures is one of looseness, which implies that expert designers have an extraordinary openness to consider multiple contexts (Goel \& Pirolli, 1992; Liikkanen, 2009). Goel (1995) explains that designers typically use a limited-commitment-mode control strategy that can enable them 
to generate and evaluate design components in multiple contexts. Since design plans and specifications are usually required to be produced in a finite amount of time and interpreted by third parties, they are required to make, record, and propagate commitments. However, as there are no right or wrong answers in designing and no real direct feedback from the world, the evaluation functions and stopping rules that designers use will be derived from personal experience and immersion in the project by a designer. Since few logical constraints exist regarding design problems, deductive inference usually plays only a minimal role in the problem-solving process. Most decisions result from memory retrieval and non-deductive inferences.

Closely connecting to this understanding is documented evidence of designers' ability to increase their consideration of design elements as part of particular solutions (Cross, 1997; Kim et al., 2007). By combining these understandings of designers loose control structure with the role that internal and external sources of information may play in the early phases of the design process, the existing theory of information processing is extended. Therefore, the focus in this article is on designers' commitment to ideas and the relationship between internal resources and situational information.

\section{Embodiment principles}

Interacting with the environment in which a design problem is situated seems to be an assumption often taken for granted and not often integrated in computational models (Oxman, 2002), and often implied in conventional information processing theories (Goel, 1995; Simon, 1996). Apart from attempts from metaphysical literature (Shani, 2012), it is, however, not common that theorists explicitly connect the two. This is what this article contributes to by connecting four prominent embodiment principles defined by Richardson et al. (2008) and implied by Anderson (2003) with information processing theory of Goel (1995). In the following section the principles of affordance, perception-action, intentionattention and specification of information are discussed.

Affordances are real, discoverable properties of the environment and objects in such environments independently of them being perceived. Researchers have thus far not been successful explaining how designers purposefully perceive such properties in the environment in order to fill the gaps in information (Gero, 1999) entering the start state of the design process. Gibson (1986) claims that affordances are neither physical nor mental. As the world and everything in it is viewed as neutral, affordance is neither an objective nor a subjective property. As such, the environment presents possibilities for action. This means that by discovering properties of the environment, designers are considered to be able to act upon it in an informed manner. For example, substances vary in size, shape, viscosity, density, cohesiveness, elasticity, and plasticity-variations. This has implications for the suitability of material chosen for a design and can therefore serve as opportunities or possibilities for action by designers. In order for designers to perceive what an environmental surface, substance, place, object, or event offers requires of them to interpret what their observations mean (Gibson, 1986).

Logically connected to the notion of affordance is that of perception, as affordance implies that properties are perceived. Affordance therefore enables designers to recognise what objects, parts of objects or properties of objects, which are part of the physical environment in which the problem task is situated, can be used to guide them in the decisions they take. The embodied view of perception is that perception cannot be understood without reference to action. Gibbs (2005) explains that people do not perceive the world statically, but by 
actively exploring the environment. Of importance to this article is the notion that perceptionaction results from the understanding that all human activity involves embodied correlations. As such, perception is an act involving perceptually guided exploration of the environment. Therefore the function of vision is seen as keeping the perceiver in touch with the environment and to guide action, and not produce inner experiences and representations. The function of vision is therefore seen as keeping the perceiver in touch with the environment and to guide action.

Closely related to the idea of affordance, and perception-action, is that of intention-attention. The latter notion aims to answer the question related to 'how it might be possible for designers to act on their perception'. The theory implies that experts know where to look for useful information and pay attention to possible physical aids, and know when to use them to facilitate their focusing on their intention, a phenomenon that Suwa and Tversky (1997) explored. This implies that designers, once they made a sketch, consider it in the same way they consider any other external object - it affords perceivable information on which they react. Goel (1995) claims that the actual presence of a sketch, also serves to change the computational loads involved in design problem-solving. However, it does not fall in the scope of this article to explain representations or intentions in detail.

In contrast to the ambiguous information often found in design briefs, the information afforded by the external environment, is specific. This means that 'information' involve patterns that uniquely specify properties of particular elements in the external world are perceived and can be recalled. Such information is specific and unambiguous (Richardson et al., 2008). This could explain why expert designers are able to compare newly perceived objects or properties thereof and associate it with properties of objects they can remember from previous cases, or personal experience. They seem to automatically compare patterns or similarities between old and new ideas. In this article, I argue that it is this specification of information that contributes to designers' ability to structure a particular design problem as the environment affords information lacking in the start state of the design process. I further argue that it is the specificity of such information that stimulates recall of knowledge of patterns, which contributes to designers generating possible solutions.

In the light of the theory embedded in the conceptual framework, and the logic connections between the various components thereof, the combination of information processing and embodiment theories, seems necessary if one is interested in identifying instances where designers apply the ability to move fluidly between internal sources and external sources of knowledge.

This conceptual framework was productively applied to study the manner in which expert designers integrate their internal processes with external information in a $\mathrm{PhD}$ research project referred to earlier. The advantage of the framework is that it acknowledges complexity; the details of which can be observed in the moment-to-moment actions of designers. However, no claim to comprehensiveness is made here and what I present here is only a snapshot of the total data sources of the original study (Haupt, 2013). Furthermore, as is the case in most protocol studies, irrespective of the framework, is that it can only analyse what can be observed in presentations of designers and cannot access mental processes that is not externally represented (Goel, 1995). A vignette of the study in which the framework was used is presented in the following section. 


\section{Case study - a vignette}

The author used the think-aloud-protocol method in which she acted as client in a quasiexperimental design study. The data consisted of two to three hours' video recorded verbal utterances and sketches produced concurrently of three separate protocol studies of expert architects, mechanical engineers and industrial designers. A mixed methodology was used and inductive-deductive reasoning cycles (Tashakkori \& Teddlie, 2009) were employed to examine the relation between existing knowledge (stored in the LTM) and situational information emerging throughout design protocols. The long term aim of such an alternative model is to assist in eliminating possible conflicting dualist approaches to design cognition and to contribute to a better insight in potential instructional strategies that may be most effective during the early phases of students' design projects.

\subsection{The design}

The author adapted the conventional concurrent TAPS protocol studies by agreeing to a 'snowball sampling' (Cohen, Manion, \& Morrison, 2007). The participants all selected thinking partners that they considered as equally expert as themselves. The architect and engineer wanted to work with one partner each, while the industrial designer chose to work with two team members. All participants agreed to work until they had arrived at an appropriate conceptual solution for the problem, allowing for a maximum of three hours. This meant that their design tasks could not be too complex. All three protocol studies entailed three distinguishable practical phases. Firstly instructions were given after which it was studied by the participants for approximately ten minutes; secondly they explored the environment for approximately twenty minutes; and finally they developed a solution in a studio close to the setting for approximately ninety minutes. The whole process lasted between two and three hours and was video-recorded by a video technician. Consent was given to use any of the data and results for educational and dissemination purposes, but using pseudonyms were unanimously agreed on. Participants were identified and selected using expertise criteria (Ericsson, 2003) and accessed through networks amongst professionals and academic colleagues. In general I accessed award winning designers in the fields of architecture, mechanical engineering and industrial design, all of whom were involved in professional practice for more than ten years.

\subsection{Experimental context}

Each of the participating disciplines received their own ill-structured design tasks, situated in its own particular socio-technological context.

\section{Architecture task}

The architects' task involved the design of an open-air theatre in the garden of the University of Pretoria, Groenkloof campus. An obtrusive existing concrete sculpture of considerable size is found in this area. The architects had the following external resources at their disposal: one document outlining the experiment procedures and another containing the design brief that motivated the need for the theatre and specified the client's needs and requirements. They inspected the site, took photographs and used colour aerial photographs of the site which they downloaded from Google Earth once in their own studio. They had access to external objects including the Internet and reference books, pens, pencils, paper and transparencies. Figure 2 shows this context of the architects. 


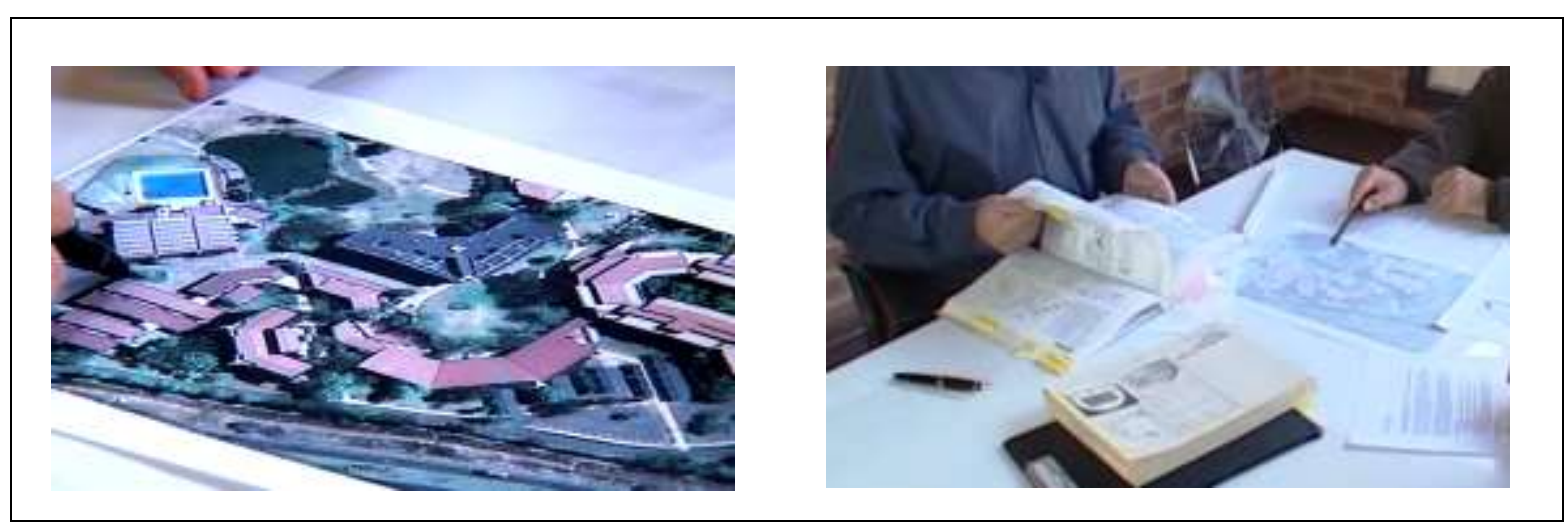

Figure 2: External resources used by Participants P-A

\section{Mechanical engineering task environment}

The mechanical engineers' task involved the design of a rotating stage for the theatre described above. However, they did not have the architects' specifications. Therefore, the engineers' task was to design a multi-purpose stage system irrespective of the size and shape the architects would commit to. This means that they had even less specific information than what is typically expected in mechanical engineering briefs (Vincenti, 1990). The implication was that their design task opened the opportunity for the engineers to interpret the need of the client in multiple ways. The participants also received two documents: an outline of the experiment procedures and a design brief that motivated the need for the theatre's rotating platform and specified the client's needs and requirements. They visited the site and made site drawings. The remainder of the experiment was conducted in a studio on the campus, within walking distance from the site. The external resources available were drawing paper, pens, pencils, reference books, the Internet, and sketches and photographs of the site. Figure 3 shows the context of engineers' setup.

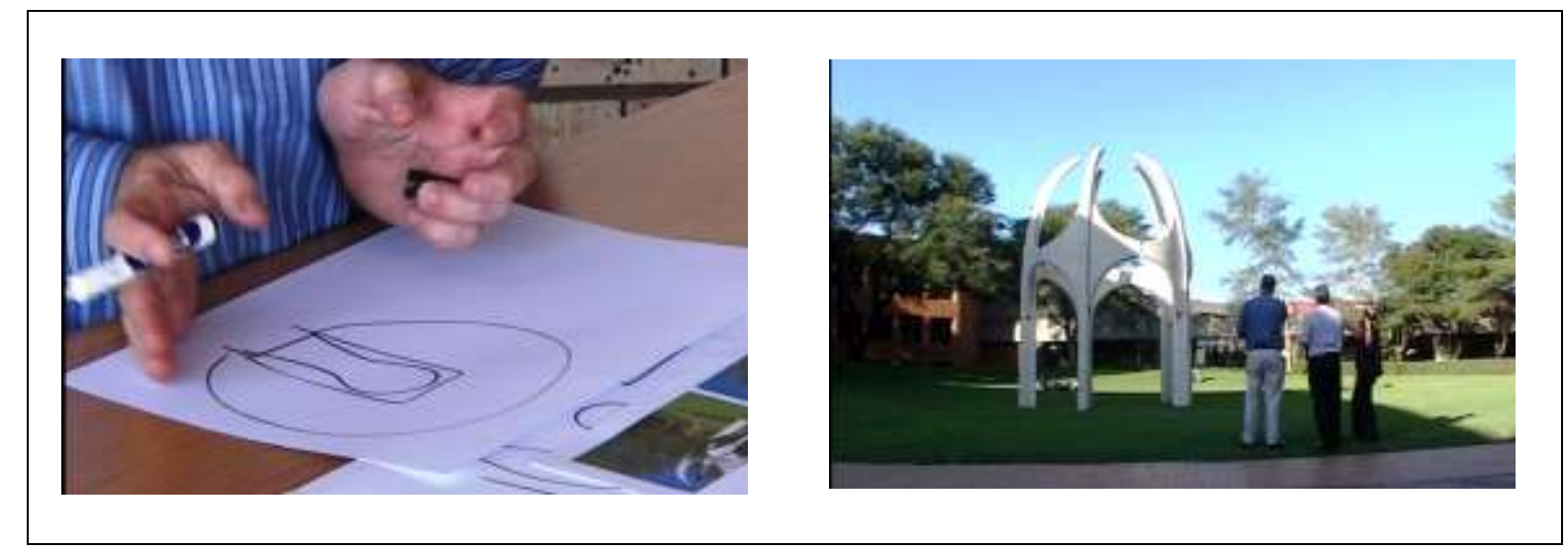

Figure 3.

External resources used by Participants P-E

\section{Industrial designing task environment}

The industrial designers' task involved the design of a device or system that could assist technology education staff in managing the use and storage of Lego ${ }^{\mathrm{TM}}$ parts on and off the campus of the University of Pretoria. These designers also received two documents as external resources: an outline of the experiment procedures and a design brief that motivated the need for the device and specified the client's needs and requirements. They further had access to a variety of external resources including examples of the said Lego $^{\mathrm{TM}}$ and existing 
packaging. The experiment was conducted in a venue similar to sites where Lego $^{\mathrm{TM}}$ is typically used. They also had access to the Internet, drawing paper, pens and pencils. In Figure 4 the multiple external sources used by the industrial designers are evident.

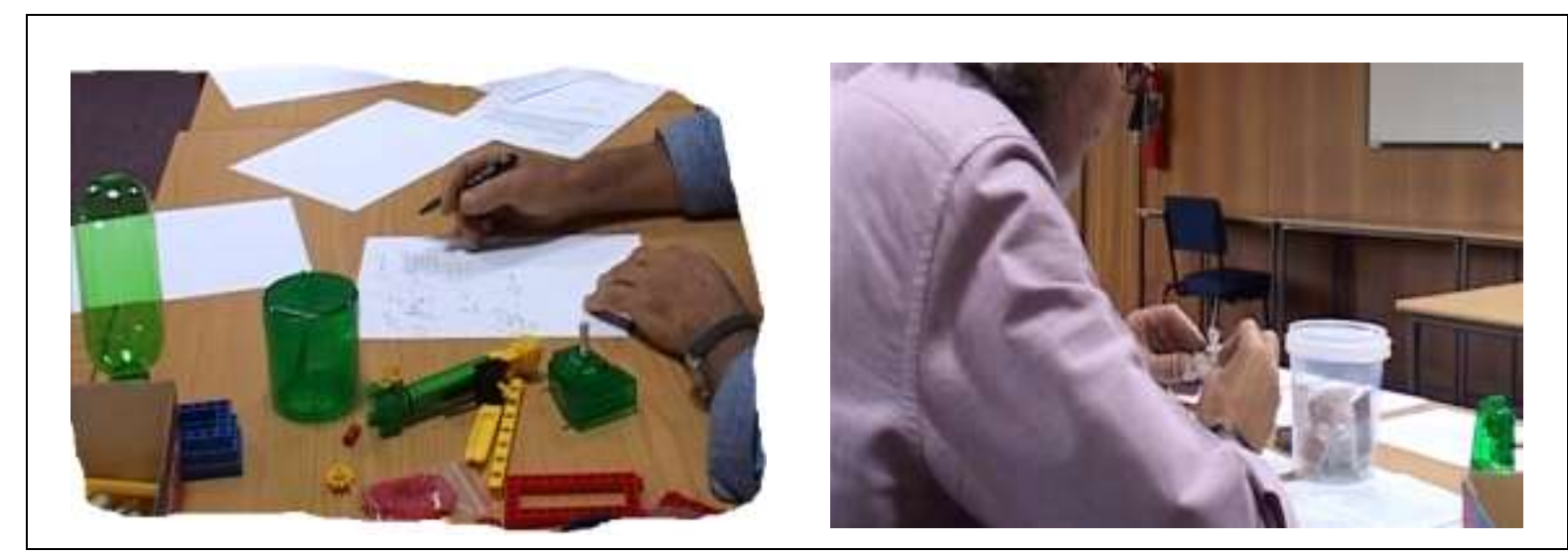

Figure 4. External resources used by Participants P-I

\subsection{Methodology and quality}

I applied a case study design using concurrent think-aloud-protocol-studies (TAPS. The conventional experimental research design was adapted conventional laboratory protocols where individuals' protocols are recorded to one where I participated as client and allowing individuals to team up with one or two partners to allow more naturalistic think-aloud dialogues. I used parallel mixed methodologies, with a dominant qualitative focus, to interpret verbal (utterances and writing) and visual (sketches and diagrams) data. This helped me to cross reference, count occurrences and measure units of time. In the qualitative part of the study credibility was achieved through internal validity ensuring qualitative rigour. The theory underlying the conceptual framework (Figure 1) guided construct validity in coding and interpretations contributing to credibility, transferability, dependability, confirmability and authenticity through triangulation, using multiple data sources and controlling observations added to trustworthiness of the results.

In the parallel quantitative part of the study descriptive statistics were used to summarise the data. Central tendency measurements such as the mean, mode and median and as measures of distribution, including temporal measurements, the ranges of counts, minimum, maximum and standard deviation were analysed and interpreted in terms of individual categories (thematic identification of embodiment principles) (Coolican, 1999). I used SPSS to generate item and group outputs. Outputs included frequency measures, standard deviation, minimum and maximum temporal indications and range. An inter-coder reliability rate of for the overall re-coding consistency for all the transcriptions was 86,5 percent. A re-coding consistency of 82 percent was achieved for coding the sketches. Quantitative validity was furthermore generated by the consistency and nature of the nature of the design tasks. The premise of the validity was embedded in the understanding that the closest connection between thinking and verbal reports is found when participants verbalise their thoughts generated during their task completion (Ericsson \& Simon, 1993).

\subsection{Data structuring}

Extensive data structuring, using guidelines by Suwa, Purcell and Gero (1998) as applied to my conceptual framework (Appendix 1), was necessary due to the large amounts of data generated by the participants' utterances and sketches. A semiotics approach was used to 
analyse the content and structure of the various outputs in order to draw cross-correlations between content, structure of thoughts, sources of thoughts and cognitive phases. This means that instances of cross-correlations between the meaning of words and pictures and the problem structuring and problem solving phases could be plot against overlapping time measurements. It further had the advantage that I could observe the sources of thoughts as well as the moment to moment development of their thoughts, leading to simplifying complex processes and mapping large quantities of data (Appendix 2) - multi-directional maps, which represents the connection the participants made between their internal processes (psychological characteristics) and external resources (embodiment principles) described further on. In the following section I provide the quantitative results of this connectionmaking process, augmented by qualitative evidence thereof.

In coding the instances the participants' relevant cognitive phases and interpreting the cognitive significance of duration of each phase well-known psychological operators observed in the utterances of the participants, namely 'commenting', 'qualifying', 'elaborating', 'justifying', 'evaluating', 'proposing' and 'repeating' (Goel, 1995) were used and linked to the sources of thoughts. Cognitive phase identification was also connected to the semantics and syntactics of sketches accompanying verbalized and visualised decisions. Activities suggesting that the participants resorted to intentional and systemic in-the-moment interactivity between the internal and the external world (Kilgour, 2006; Smith, 2005). People, including partners and client/researcher as well as activities, such as talking, writing, sketching and gesturing counted as external resources involved. Close observation of what was happening during each verbal utterance recorded on video, made it possible to distinguish when the participants' actions were triggered by their design briefs or information provided by the client (researcher). I could also determine and code when partners in the various groups made sketches.

In the verbal utterance of the industrial designers, I could, as an example of such close observation derive the cognitive phase, the psychological characteristic and the relevant embodiment principle from the utterance quoted in Excerpt 1.

\section{ADRIAN 1:02:07}

\section{Excerpt 1}

The fourth idea I had, was potentially you could make up what we call vacuum formed trays, I could call them vacuum kit trays and each vacuum formed sheet has spaces for specific components, they, everything has it place and it must fit into that vacuum formed tray. Then you could make different kits up and then you could stack them all together, one on top of the other and when you hand it out it is very visible everybody can see what is in each kit and everything has a place where it can fit back, so the vacuum forming is a fairly easy and inexpensive way, and you're using a clear film then the parts can be very visible and you could maybe have a rack we you can stack them all, uhm as a solution and the maybe involve the user quite a lot in having to take parts out and put them back in at the end and hand back. Receive a full tray and hand out and hand back full trays of bits and pieces back in their places.

The 'fourth idea' (Excerpt 1) was generated in quick succession of a previous 'third' idea and pointed to the cognitive phase of preliminary problem solving. This could be inferred from the relative vague outline of the idea of 'vacuum formed trays'. The lack of physical detail, and focus on functionality and behaviour confirmed this cognitive phase. The psychological 
characteristics identified in this utterance were coded as 'transformation', 'evaluation' and the 'use of multiple symbol systems'. As the utterance was accompanied by a series of rough sketches by the participant, it was interpreted as evidence of both transformation and the use of multiple systems. Application of the participant's evaluation function was derived from his judgment of 'so the vacuum forming is a fairly easy and inexpensive way,...' and 'the parts can be very visible ...'. Finally the ecological principle of intention-attention was inferred from the participants focus on functional, behavioural and implementation intentions. Functional intention was derived from 'each vacuum formed sheet has spaces for specific components', behavioural intention from 'you're using a clear film then the parts can be very visible...' and implementation intention from 'Then you could make different kits up and then you could stack them all together...'. Resulting from microscopic close reading of the verbal utterances of all the participants' entire protocols and their accompanying sketches, the prominent findings are discussed in the following section.

\subsection{Findings}

In this section I present some of the quantitative results and verbal evidence firstly related to the participants' cognitive phases and secondly to internal-external interactivity.

\section{Distinct cognitive phases}

As stated earlier, problem structuring and problem solving phases sometimes overlap and it is not always clear where the one phase ends and the other starts. I call this overlap a 'leaky phase' (after Goel and Pirolli's (1992) notion of a 'leaky module').

Unambiguous problem structuring instances were characterised by the process involved when the participants read their design briefs, asking questions that flowed from reading and figure implications of the requirements of their tasks. An example of such structuring is evident from Excerpt 2 (architecture participants' protocol).

\section{Excerpt 2}

\section{ANDREW 00:50:04}

So that we can just get a feel for ... on the time ....

The look and feel should convey the concept of creativity, growth and playfulness'.

So this should already be out of the box, you know - how this thing will work ... but can we question this?

JONATHAN 00:53:34

Textual constraint'...That's why I asked if this was people or what? 'Audience not being exposed to looking in the sun while watching the performance.' That's why I asked if this refer to people or what? So this thing should face south.

From Excerpt 2, the author could deduce that the instances that the participants spent on structuring their design problems could also be linked to their explicit decomposition methods. This inference is supported by studies which suggest that experts rely on a 'dualmode' of decomposition (Ho, 2001; Liikkanen, 2009). This means that they use explicit decomposition strategies at the beginning of their process as can be seen in Excerpt 3.

\section{Excerpt 3}

BRIAN 00:04:28

OK, what you have given us here ... would this be sort of an average would you say, 
like, $u h m \ldots$

BRIAN 00:04:39

In other words, I see one green bottle, but I see quite a lot of red...

BRIAN 00:05:09

Individually? Or in sets?

CLINT 00:05:21

These teaching sessions that are conducted by the relevant lecturers... Uhm, are they specific to the subject? In other words they will take a Lego set which is physics orientated or take a Lego set which is mechanically engineering orientated.

BRIAN 00:06:17

OK, so let's just go over this again.

I found explicit attempts in the participants' problem solving phases and, as sub-goals emerged, that they used explicit and implicit methods of decomposition as seen in Excerpt 4 (engineering protocol).

\section{Excerpt 4}

\section{EDWARD 01:35:50}

Then you have here ... this is the inside ... the stage goes that way, say to there. And here you have a whole rotating axis ... Okay. Sure.

Question is then about maintainability'.

EUGENE 01:36:07

That's easy - easy to maintain.

EUGENE 01:36:41

You've got a motor - there - where ever that motor sits .... You put it ... on the platform ... you make a handle for it ... You can put it any place.

EUGENE 01:36:20

Yes ... and ... you can put your wheels .... It depends ... at this moment that thing is not much higher - I don't know what the incline is - it cannot be much more than 200-300 mm. So, it is relatively flat. So you can easily - if you open the platform, you can reach it to change the wheels, or whatever.

Put the motor anywhere. Put it there - with access from there. Open the door and put the motor there. You can access it - you let the wheels go round-at the backit doesn't matter.

EUGENE 01:36:54

And underneath this platform you will have an electric box which you can open. Then you can have all your electricity ... because you will have some kind of other wiring to the platform in order to have power on the platform.

In contrast, 'leaky phases' contained elements of problem structuring and problem solving in the same modules. Leaky instances often occurred as a result of the emergence of subproblems and the generation of conceptual solutions. The participants partly solved some of the sub-goals at the point of emergence, while others were solved at different times during the explicit problem solving phases, whereas a number of sub-goals were ignored. Excerpt 5 illustrates how the engineers in the same module were making a decision as they raised a question to further structure their problem, suggested an approach, questioned their suggestion and delayed making a decision. 


\section{Excerpt 5}

EUGENE 01:27:15

Our engineering therefore only functions as far as the rotating platform. Up and down?

EUGENE 01:31:36

I don't think you want to go much higher than what is level with the highest point of the ground.

EDWARD 01:31:41

I mean in terms of the hole - to lift the whole floor up and down like a shaft. I wonder if it makes any sense for this purpose. It is too 'fancy. I don't know-you can say. It is too fancy, keep it simple.

Derived from the structure of the participants' verbal and visual representations, as well as from the content thereof, the distinct cognitive phases of the participants' thinking process could be derived. The frequency calculations of the proportion of time each groups spent on each of the phases calculated from the time (per seconds) proportionate to each group's total time used to complete their tasks, is summarised in Table 1.

Table 1 Quantifying the problem solving space: Summary percentage of overall temporal structure of the cognitive phases found in the TAPS across participants

\begin{tabular}{|c|c|c|c|}
\hline & \multicolumn{3}{|c|}{ PHASE } \\
\hline Design group & $\underset{\%}{\text { Problem structuring }}$ & $\underset{\%}{\text { Problem solving }}$ & $\begin{array}{c}\text { Leaky phases*** } \\
\%\end{array}$ \\
\hline Participants P-A (Architects) & 33 & 31 & 12 \\
\hline $\begin{array}{l}\text { Participants P-E } \\
\text { (Mechanical engineers) }\end{array}$ & 31 & 33 & 16 \\
\hline $\begin{array}{l}\text { Participants P-I (Industrial } \\
\text { designers) }\end{array}$ & 23 & 28 & 10 \\
\hline
\end{tabular}

(*Proportion (\%) Time period (seconds))

(**Goel \& Pirolli (1992) defines a 'leaky' phase as those instances where problem structuring and problem solving overlaps)

This information suggests broad patterns in the dynamics involved on a macro level. However, on a micro level, it was found that each of the groups had different ways and sequences of activities in decomposing their problems. The qualitative coding was subsequently transferred into a binary coding system. Applying SPSS software to the coded transcripts, the distribution of instances across the entire protocols of each of the participating design teams is visualised in the boxplot in Figure 6. 


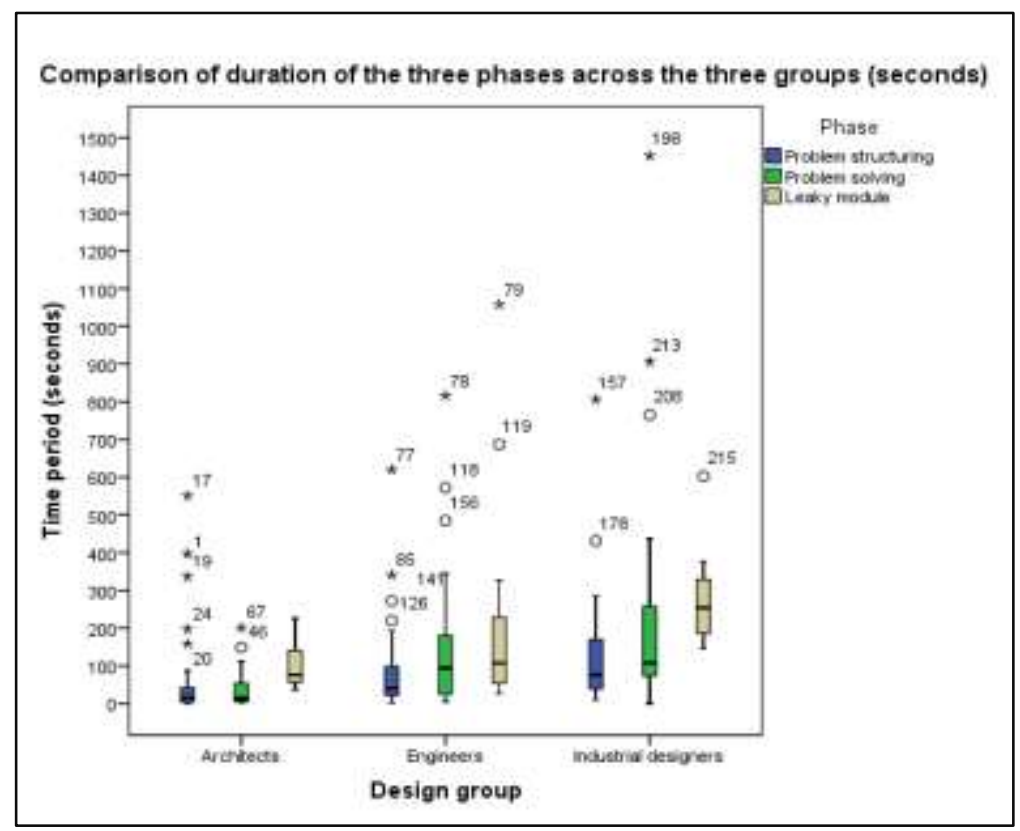

Figure 6 Distribution of instances across the protocols of each of the participating design pairs/teams

It does not fall within the scope of this study to expand on the detail of each of the participants' micro processes leading up to the mapping. However, what is of interest here is the way in which the participants integrated their access to embodied information with their internal processes.

\section{Internal-external synergy as psychological characteristic of expert design cognition}

For the two cognitive phases, problem structuring and problem solving, of each of the participants, I individuated modules within which the psychological characteristics (Goel \& Pirolli, 1992) 'control structures', 'personal stopping rules', and 'evaluation functions' occurred simultaneously with observations of the use of types of knowledge. As such, relating the embodiment element intention-attention instances with types of knowledge could indicate which type of knowledge was most prominent in the selected psychological features observed. It is important to note that I did not differentiate between participants' references to the artifact that they were designing and references to other artifacts in the environment or stored in their memories when assigning values to their knowledge categories. For the purpose of this discussion I present the frequency counts and present graphs that demonstrate which type of internalized knowledge interacted with each of the afore mentioned embodiment principles proportionately (percentages) when the individual participating teams applied psychological strategies which included control structures, personal stopping rules and evaluation functions in their problem spaces in each of the cognitive phases. As explained earlier, I focused on these three characteristics as I considered them as essential to problem structuring, decision making and incremental development of ideas.

Each of the combinations was in turn combined with the embodiment principle intentionattention only as I considered it as essential to linking the information processing assumption of intention, to what designers are attending to at a particular instance. Furthermore, relating intention-attention instances with types of knowledge could indicate which type of 
knowledge used by participants was most prominent in the selected psychological features observed.

\section{Architecture participants}

Figure 7 summarises the number of knowledge types applied by Participants P-A in their problem structuring phase. Through the association of knowledge with their intentionattention instances while applying control strategies, personal stopping rules and evaluation functions, I gained insight into the dynamic interaction between their external and internal cognitive processes.

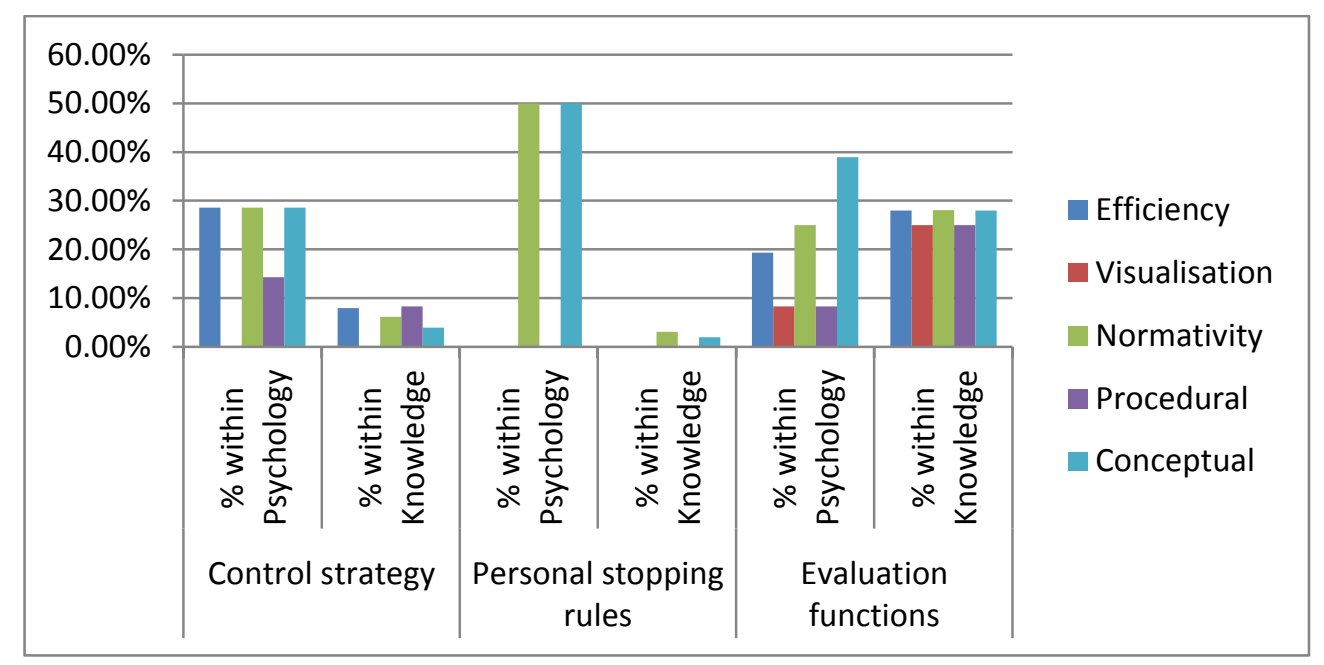

Figure 7 Interaction between Participants P-A's knowledge types, external and internal processes in their problem structuring phase

In Participants P-A's problem structuring phase, when they applied their knowledge in control strategies, they relied primarily on procedural knowledge, conceptual knowledge and efficiency knowledge. Their normative knowledge also featured proportionately high in their control strategies. What was interesting though was that at this stage of their process, Participants P-A did not generate any ideas through visualisation as control strategy but they rather used their sketches to support their personal stopping rules. They also used their sketches to illustrate the design principles they normally apply when judging the social and spatial effectiveness of objects in space involved in urban design normatively. They visualise these abstract principles by making rough sketches using external primary shapes and spatial relations of comparable design cases.

During their problem solving phase, (Figure 8) when generating ideas for their open-air theatre, Participants P-A used their efficiency knowledge as a mechanism to apply their control strategies. It seemed that they used their knowledge of efficiency during the problem solving phase primarily to guide their control strategies when they made their decisions. This implies that efficiency did not play a significant role when they indicated their personal preferences and evaluated ideas normatively, but were rather elements that affected their ideas of how to create the most suitable atmosphere and general aesthetics of the theatre that they were designing. 


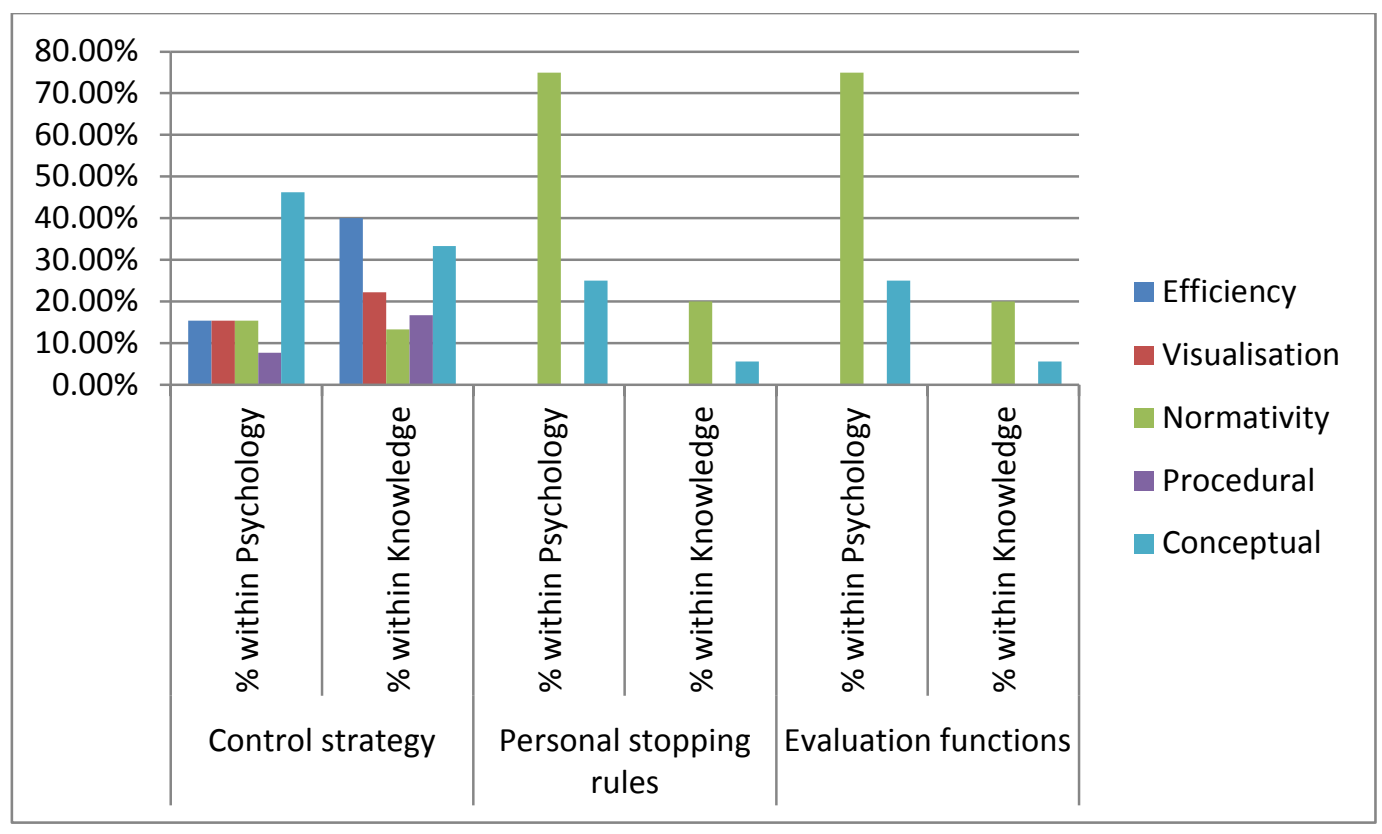

Figure 8 Interaction between Participants P-A's knowledge types, external and internal processes in their problem solving phase

There was a steep increase in the architects' use of external visualisation during their problem solving phase when combined with all the relevant psychological characteristics and embodiment principles. It seemed that they primarily used their sketches as a means to represent their control strategies. They made a relatively large number of normative judgments simultaneously with sketching and applying control strategies. This means that their sketches served as tool to express their personal stopping rules and evaluation statements. Coinciding procedural knowledge and the application of control strategies in order to solve their problem was less than when they were structuring their problems. Little overt evidence was found of them using procedural knowledge concurrently with instances of personal stopping rules and evaluation functions. In turn, when they developed their solution ideas, the architects constantly used their conceptual knowledge of architectural artifacts, their structural and physical qualities, and their social and cultural functions in urban design situations in general, and specifically in university campus situations.

When Participants P-A applied their control strategies, a co-occurrence of efficiency, visualisation, normative, procedural knowledge and conceptual knowledge, was observed. This suggested that there was a complex layering of the knowledge types when the participants exercised control. The absence of efficiency references when they used their own personal stopping rules suggests that they used other norms in their evaluation functions than efficiency norms. The absence of visualisation as means to represent their personal stopping rules during their problem solving phase, suggested that they used their sketches to generate new ideas and not as a way of expressing their personal preferences.

\section{Mechanical engineering participants}

The statistical data of Participants P-E's problem structuring phase is presented in Figure 9, which indicates that they had a different strategy when they used various knowledge types during intention-attention instances compared to their problem solving phase. 


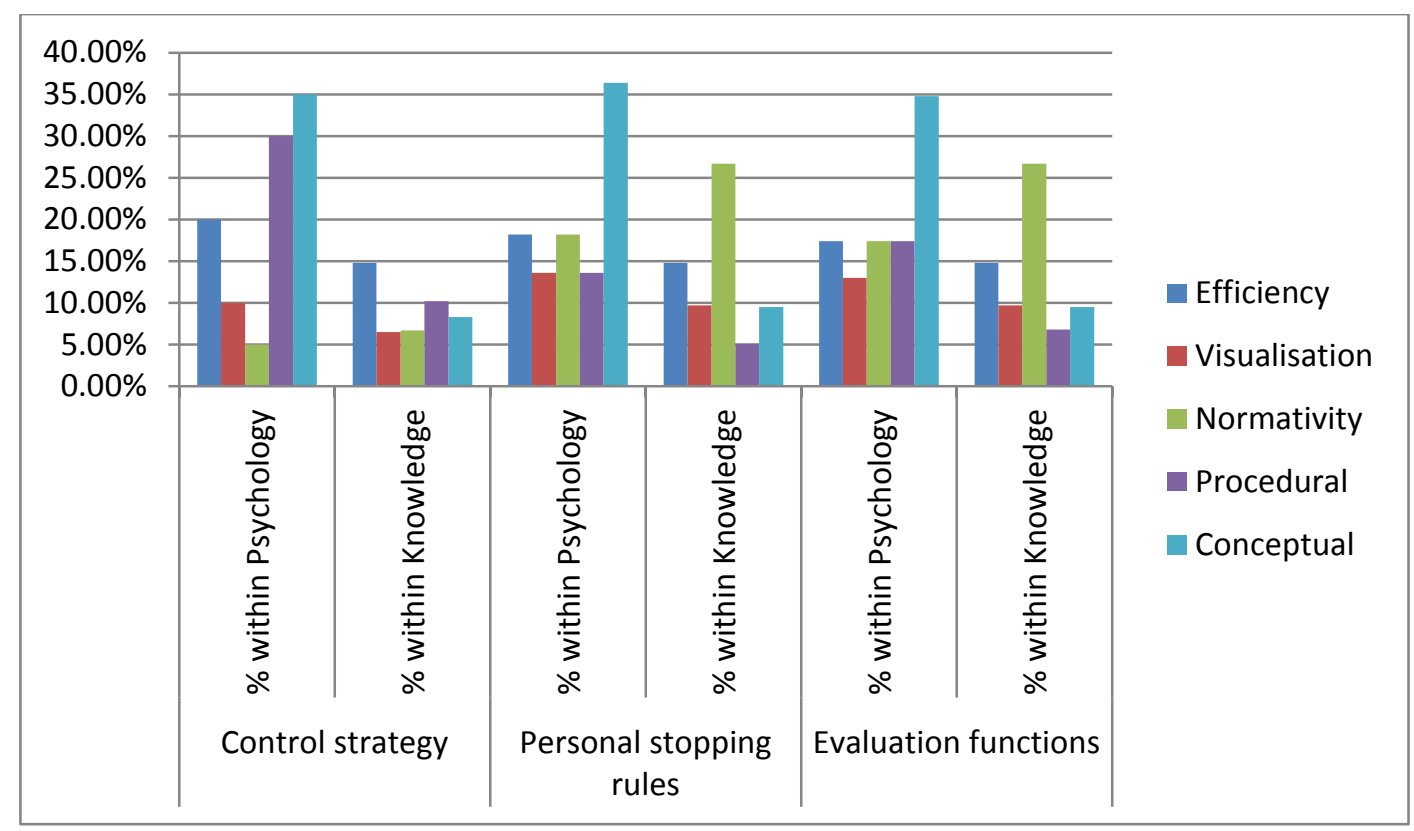

Figure 9 Interaction between Participants P-E's knowledge types, external and internal processes in their problem structuring phase

Comparing Participants P-E's problem solving activities with their problem structuring activities that I observed of control strategies, personal stopping rules and evaluation functions, the statistical data yielded the information presented in Figure 10.

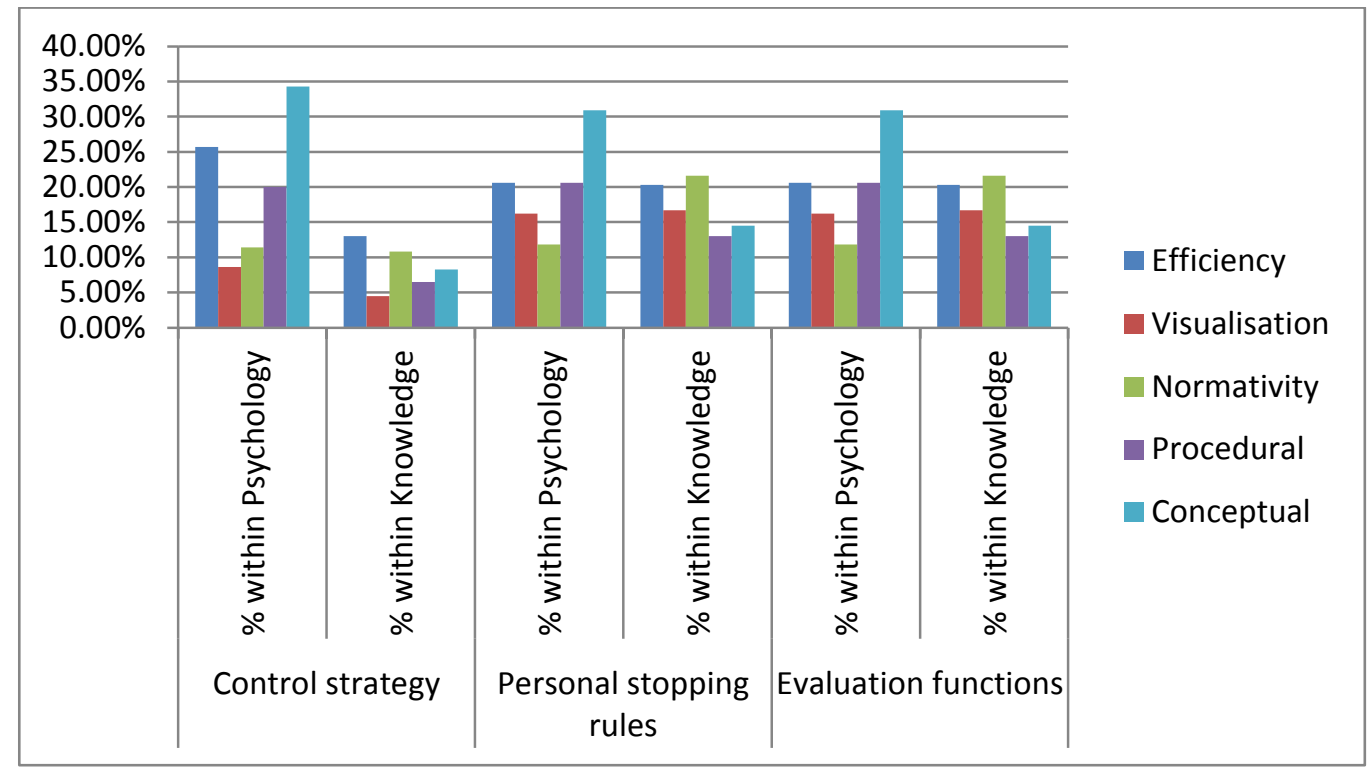

Figure 10 Interaction between Participants P-E's knowledge types, external and internal processes in their problem solving phase

During the problem structuring phase, the engineers attempted to understand how and where the mechanically driven theatre platform should be standing and whether they should incorporate the existing sculpture on the site. As was the case with the architects, the engineers consistently used efficiency knowledge when they applied control strategies, personal stopping rules and evaluation functions concurrently with intention-attention instances. Their relative low counts of use of procedural and conceptual knowledge during 
problem structuring. They tended to find information through questioning and observation rather than apply their internally stored knowledge. However, they made relatively high percentage (26.70\%) normative judgments, not when applying control strategies, but rather that was embedded in their personal stopping rules and evaluation judgements. The engineers did not use their knowledge to express intentions. I thus inferred that they had not fixated on any preconceived intentions at the time. This confirmed their openness to objective, critical consideration of perceived information. P-E also consistently produced rough sketches, although less than the architects, to support the relevant psychological processes, as were the case with the architects. This seemingly had a low influence on their number of control strategies, personal stopping rules and evaluation functions. During their problem solving phase, when the engineers applied control strategies, it was consistently co-occurring with their use of efficiency knowledge.

When they used their efficiency knowledge, there was an increase in use of efficiency when they simultaneously applied personal stopping rules and evaluation functions. It seemed that they specifically preferred to consider efficiency issues when they apply personal stopping rules and evaluate artifacts. This might be attributed to a domain related habit of thinking in this manner about artifacts (Vincenti, 1990). They used sketches increasingly during their problem solving phase to express their personal stopping rules and justify their evaluations when making choices. This co-occurred with their increased use of normative knowledge when they controlled their choices during the process of developing their artifact design. However, in the bigger picture $10.8 \%$ is a relatively small proportion of their total cognitive activities. This implied that their control strategies were loose, which opened their minds to alternative ideas, a notion that is widely acknowledged in expert design literature (Cross, 2007; Visser, 2004). The engineers' number of normative statements that coincided with their efficiency knowledge when applying personal stopping rules and evaluations function confirmed my previous idea that they used efficiency as personal norm when they evaluated design quality. There was fifty percent increase in Participants P-E's use of procedural knowledge. This coincided with their personal stopping rules and evaluation functions in the problem solving phase, which I interpreted as a personally bias of the engineers towards implementing particular procedures when they designed artifacts. The examination of the details of these procedures however, did not fall within the scope of this article.

\section{Industrial designing participants}

In Figure 11, the statistical data of Participants P-I's problem structuring phase indicates a strong, one sided use of knowledge. This trend primarily supports their evaluation functions which did not feature as support of their control strategies and personal stopping rules when they structured their design task. It is evident that they did not make any normative judgments at this stage and did not commit themselves to any procedural knowledge. They did, however, make relatively extensive use of their visualisation knowledge, as seen in the numerous sketches that they made. Interestingly, their visualisations primarily coincided with their evaluation functions, which accounted for $40 \%$ of the intention-attention instances coinciding with Participants P-I's evaluation functions. Against the background of the industrial designers' systematic break down of their interpretation of the design problem during the problem structuring phase. I reason that the industrial designers used their visualisation knowledge at that stage primarily to externally support testing of their conceptual knowledge of the physical properties and potential uses of alternative artifacts. This assumption is supported by the lack of external visualisation activities that coincided with control strategies and personal stopping rules. In addition, the extensive written documentation of their evaluation of advantages and disadvantages of ideas seemed to 
function as additional external support of Participants P-I's visualisations. The trends discussed here are reflected in Figure 11.

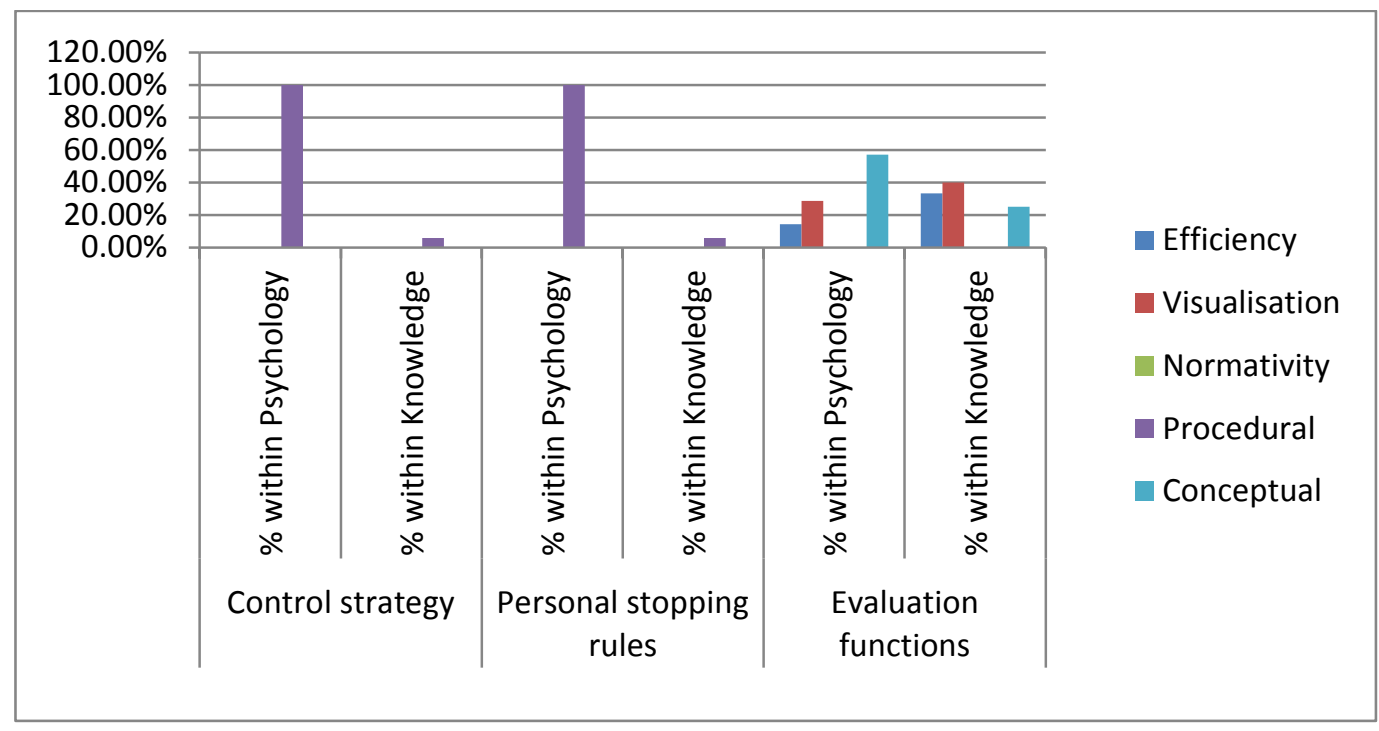

Figure 11 Interaction between Participants P-I's knowledge types, external and internal processes in their problem structuring phase

When they were solving their design problem, Participants P-I's statistical data indicated that they applied efficiency knowledge to a limited extent in combination with control strategies, but primarily used this to support their evaluation functions, while it was absent when they applied their personal stopping rules. This implied that they, in general, entertained their own personal stopping rules relatively infrequently when using any of the various knowledge types. They engaged in the application of control strategies and in particular evaluation functions. The relatively high proportion of conceptual and procedural knowledge that Participants P-I used when evaluating issues, lead me to conclude that they consciously and subconsciously used a cognitive strategy of applying objective evaluation functions instead of personal stopping rules when they developed a solution for their design task. Ascertaining whether the industrial designers applied objectivity consciously or subconsciously, did not fall within the scope of this article. The lack of visualisation together with low counts of use of normative, procedural and conceptual knowledge when Participants P-I engaged in control strategies indicates their limited use of control structures when developing their design problem in general. This pattern suggests their loose control structure and adaptability, which was supported by the relatively large number of alternative ideas that they visualised and subsequently evaluated without fixating too early on one particular idea Figure 12 presents the trends discussed here. 


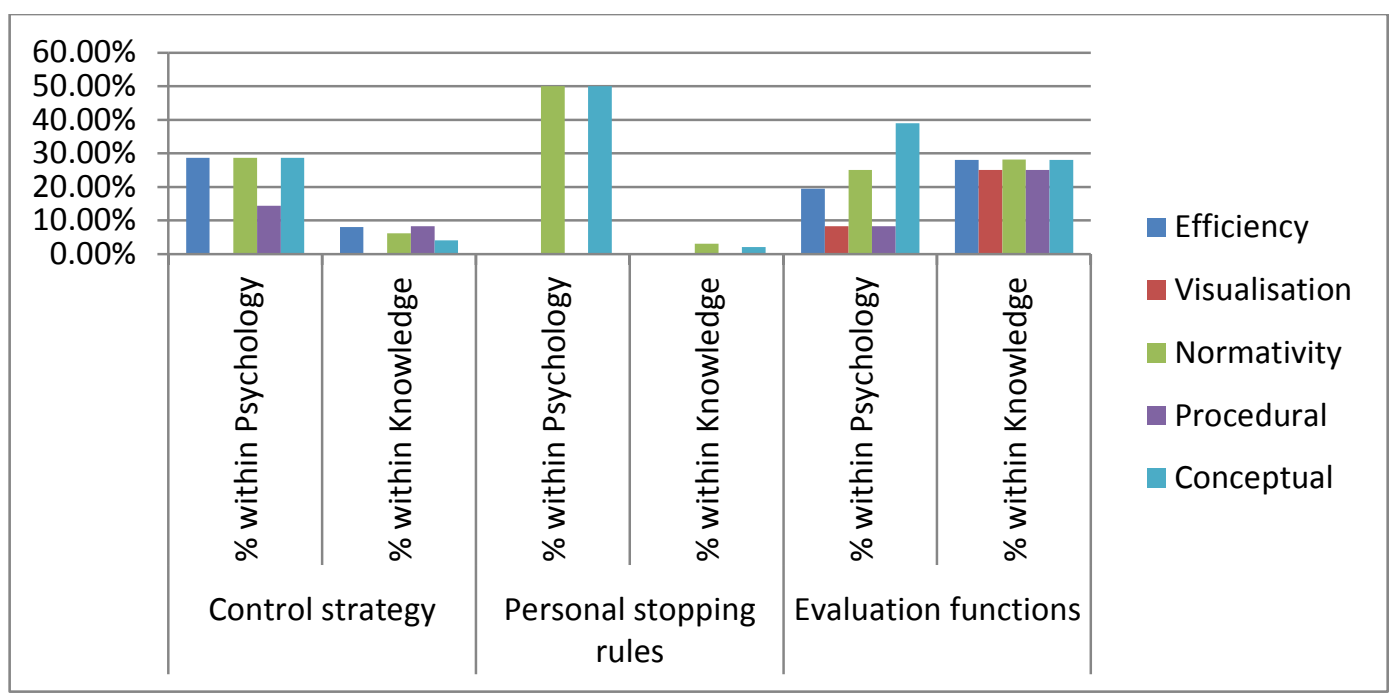

Figure 12 Interaction between Participants P-I's knowledge types, external and internal processes in their problem solving phase

From the statistical data presented in the tables and graphs above, I deduce that, during the problem structuring phases, the participants' intention-attention behaviour could primarily be associated with their use of visualisation and normativity knowledge use. In the case of Participants P-A, it can be inferred that the participants in the majority of cases, when they intentionally paid attention to something, used procedural knowledge and sketches when they applied control strategies. This observation subsequently lead to the emergence of a research question with regard to the possible relationship between sketching ideas and knowledge of procedures of implementation plans in order to achieve aspectual and functional intentions, which is not discussed in this article.

\section{Discussion}

From experts we learn that designing is an uncertain and ill-structured activity, which needs problem structuring through investigating and problem solving through designing and visualising, applying evaluation functions and communicating and more visualising, before a final product can be manufactured. This is a non-linear process requiring constant movement between internal sources of knowledge and emerging external information. I have observed that, during this interactive process, expert designers fluidly and synergistically draw interrelations between multi-disciplinary knowledge and basic knowledge types through a systemic cyclic process of perceiving specific information afforded by the external world, including their sketches, on which they react. This is made possible by designers' relatively loose control systems, personal stopping rules and evaluation functions. The end result of the cognitive change taking place in designers is essentially one of moving from a state of uncertainty to a state of certainty when they are ultimately able to present ideas in detailed specifics about potential solutions for socio-technological design problems as integrated and synthesised that can change the quality of the lives of particular people.

The case study described earlier, also showed that it is possible to gain relative direct access to what and how designers think. This means that verbal material as well as the sketches that are produced concurrently serves as data about their cognitive processes and should be included in teachers' assessment of the cognitive phases of students, instead of merely assessing the content of their ideas and quality of the end products (artifacts) of their thinking. I further assume that the ill-structured nature of design tasks require students to access information from various sources at their disposal, including the design brief (which 
was underspecified), the client/teacher (underspecifying information), long term memory relying on stored generic experiential and domain specific knowledge stored, and information embodied in the environment.

\subsection{Challenges in technology classrooms}

The implications for the lecturer are on the one hand that, whether projects are group oriented or individual, the lecturer facilitates, through scaffolding, recognition of prior knowledge and cultural diversity. This means that taking responsibility for own actions is a top-down controlled situation closely coupled with time management and continuous assessment opportunities. Control in the classroom therefore means that students' perception of individual responsibility should be encouraged, which impact on their evaluation functions, commitment to their own decisions and ultimately on students' time management skills. Furthermore, as novices, most of the students will have rudimentary visualisation and manufacturing skills rather than be experienced visual thinkers. This may impact on how confident students are to design products that are too complex to visualise or manufacture. The implications for the students, on the other hand they have to stay close to the procedures, dead-lines and individual sub-tasks involved in each of the implied roles of the students, but on the other hand be allowed to think in a non-linear, adaptive way, which mirror that of experts.

\subsection{Appropriate pedagogical approach}

On the whole I can point out that project-based learning as pedagogical approach provides an appropriate backdrop against which real-world design abilities could be fostered. This idea is gaining momentum in technology education ( Petrina, Feng, \& Kim, 2008). The reason for this is that project-based learning as explained by Savin-Baden (2007) provides the opportunity for lecturers/teachers to be involved in authentic processes of problem solving through design work as the task setter and supervisor of the projects. This is necessary as technology students are considered as inexperienced novice designers, and therefore in need of close guidance and supervision. There are more reasons for advocating the adoption of a project-based approach to developing cognitive abilities of novice designers that reflect those of expert designers. One prominent argument is that it entails a comprehensive approach to classroom teaching and learning that is designed to engage students in investigation of authentic problems (Blumenfeld et al., 1991), which connects comfortably with what I have said about the nature of design problems.

In Table 2 I present a summary of expert psychological characteristics adapted from Goel and Pirolli (1992), mapped onto extended cognitive activities for students and subsequent cognitive aims which could serve as guidelines for teachers to structure their instructional design of design projects in technology classrooms. The overall assumption is that the design problems novices receive from their teachers are relatively ill-structured and complex.

Table 2 Guidelines for cognitive activities developing psychological characteristics that mirror expert problem solving space characteristics

\begin{tabular}{|l|l|l|}
\hline Expert problem solving space & Cognitive activities & Overall cognitive aims \\
\hline Problem structuring & $\begin{array}{l}\text { Identifying missing information } \\
\text { Identifying, accessing information } \\
\text { internally and externally and applying it } \\
\text { appropriately to fill gaps in missing } \\
\text { information }\end{array}$ & $\begin{array}{l}\text { Moving from a state of } \\
\text { uncertainty to a state of } \\
\text { certainty }\end{array}$ \\
\hline Distinct phases in design & Record thinking processing, using & Taking control and \\
\hline
\end{tabular}




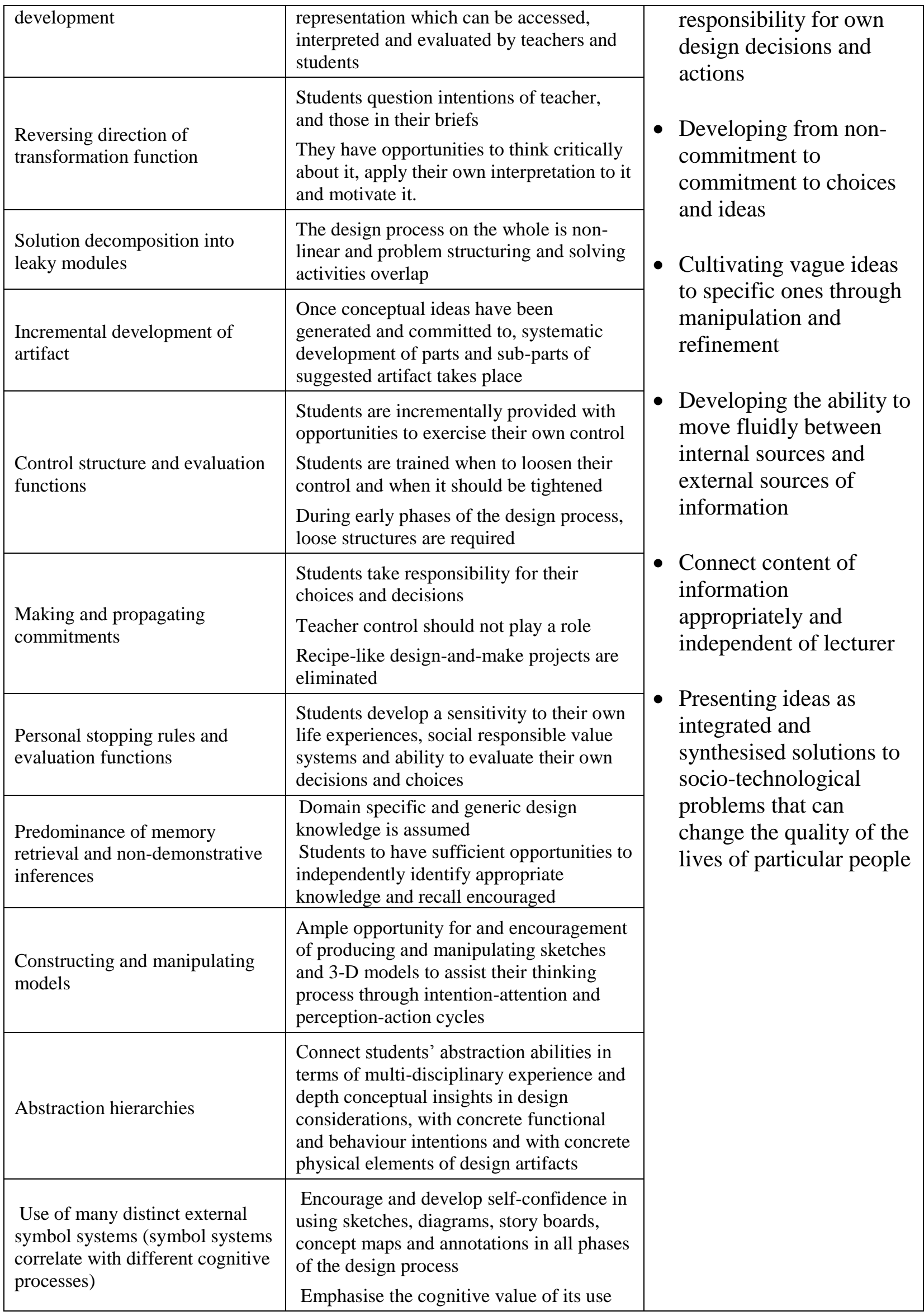


The author believes that it is more productive to integrate these theories. On the one hand abstractions and generalisation with its subsequent disregard for concrete situational influences on individuating problem solving (Kirsh, 2009), which is typical of computational theory, limit the learning experience. On the other hand, overemphasis of situational theories to provide guidance for educational programmes constitutes a narrow focus on the detail of a specific design situation without considering typical design situations. This implies that teaching programmes that by nature rely on generalised models that guide uniform and regimented computational criteria for successful learning output might benefit from a unified theory of design cognition which is amenable to generalisation without compromising individual creativity and the personal design styles of learners. Conscious planning and instilling a balanced and synergetic use of both internal resources and external information could contribute to educators keeping track through formal and informal assessment of where the dominant control for particular cognitive activities are situated.

\section{Conclusion}

If the ability to adapt one's behaviour in terms of the particular intentions and information emerging from the environment interact with each other in a dynamic manner, it may assist in establishing how designers and students adapt their behaviour from moment to moment according to changes in the problem solving situation (Ericsson \& Simon, 1993). Thus, the argument in this study is that designing is a process experienced within the environment which a designer encounters through the context of a design task. Accounting for the environment entails that accessing information cannot be limited to the internal components of a design task at hand but has to take the whole environment in which the task has to be integrated in the transformation process. Such insight into designing implies the notion that 'where you are, when you do what you do, matters' (Clancey, 1997). Therefore in order to develop complex and dynamic thinking skills of novices that mirror those of expert designers, one sided approaches of teaching and learning designing, which are promoted by separating computational and ecological theories, should be prevented. Such a balance could be struck by considering the conscious nurturing of adaptive control structures, personal stopping rules and evaluation functions of students. This could be achieved by encouraging students' intentional accessing internal resources, developing their psychological characteristics typical of design behaviour, applying various knowledge types and promoting students' interaction with authentic external elements that contextualise their design projects. Amongst other things, it implies that authentic ill-structured design tasks with authentic external elements should be integrated with the development of mental skills and resources. This could be achieved by mapping a balanced set of learning and design experiences, by considering the following:

- How much control might be found in students' internal processes, directed by internal resources?

- How much control might be found in the learning and design task environment?

- How is students' perceptual sensitivity for external influences through affordances, cues and contextual constraints built up over time?

- How is students' mental and physical flow of movement between their inner and outer world developed?

- How central is students' use of a variety of symbol systems during the early phases of their design process?

- How sensitive are teachers to the cognitive role of students' various modes of output to inform them about how much learners understand about the sociotechnological problem they are required to solve? 
- How do teachers use learners' external representations to inform them about the distinct phase in their design development?

\section{Reference list}

Anderson, M. L. (2003). Embodied Cognition: A field guide. Artificial Intelligence, 149(1), 91-130

Barak, M., \& Hacker, M. (2011). Fostering Human Development through Engineering and Technology Education (Vol. 6). Rotterdam: Sense Publishers.

Bickhard, M. H. (2008). Is Embodiment Necessary? In P. Calvo \& A. Gomila (Eds.), Handbook of Cognitive Science: An Embodied Approach. Amsterdam: Elsevier Science.

Blessing, L. T. M., \& Chakrabarti, A. (2009). DRM, a Design Research Methodology. Dordrecht: Springer.

Blumenfeld, P. C., Soloway, E., Marx, R. W., Krajcik, J. S., Guzdial, M., \& Palincsar, A. (1991). Motivating Project-Based Learning: Sustaining the Doing, Supporting the Learning. Educational Psychologist, $26(3$ \& 4), 369-398.

Brandsford, D., Brown, A., \& Cocking, J. (2000). How People Learn: Brain, Mind, Experience and School: Expanded Edition. Washington: National Research Council.

Christiaans, H., \& Venselaar, K. (2005). Creativity in Design Engineering and the Role of Knowledge: Modelling the Expert International Journal of Technology and Design Education, 15, 217 - 236.

Clancey, W. (1997). Situated Cognition: On Human Knowledge and Computer Representation Cambridge, UK: Cambridge University Press.

Cohen, L., Manion, K., \& Morrison, K. (2007). Research Methods (6th ed.).

Coolican, R. (1999). Research Methods and Statistics in Psychology (3rd ed.). London: Hodder \& Stoughton.

Cross, N. (1997). Creativity in Design: Analyzing and Modelling the Creative Leap. Leonardo, 30(4), 311-317.

Cross, N. (2004). Expertise in Design: an Overview. Design Studies, 25(5), 427 - 441.

Cross, N. (2007). Designerly Ways of Knowing. Basel: Birkhauser Verlag AG.

de Vries, M. J. (2005). Teaching about Technology. An Introduction to the Philosophy of Technology for Non-philosophers. (Vol. 27). Dordrecth: Springer.

de Vries, M. J. (2006). Technological Knowledge and Artifacts: An Analytical View. In J. R. Dakers (Ed.), Defining Technological Literacy. Towards an Epistemological Framework. New York: Pelgrave MacMillan.

Ericsson, K. A. (2003). The Search for General Abilities and Basic Capacities: Theoretical Implications from the Modifiability and Complexity of Mechanisms Mediating Expert Performances. In R. J. Sternberg \& E. L. Grigorenko (Eds.), The Psychology of Abilities, Competencies and Expertise. Cambridge: Cambridge University Press.

Ericsson, K. A. (2006). Protocol Analysis and Expert Thought: Concurrent Verbalizations of Thinking during Experts' Performance on Representative Tasks. In K. Ericsson, N. Charness, P. J. Feltovich \& R. R. Hoffman (Eds.), Cambridge Handbook of Expertise and Expert Performance (pp. 223 - 242). Cambridge, UK: Cambridge University Press.

Ericsson, K. A., \& Simon, H. A. (1993). Protocol Analysis. Verbal Reports as Data (Revised Edition ed.). Massachusetts: Massachusetts Institute of Technology.

Gero, J. S. (1996). Creativity, Emergence and Evolution in Design. Knoweldge-Based Systems, 9(7), 435-448. 
Gero, J. S. (1999). Constructive Memory in Design Thinking. Paper presented at the Design Thinking Research Symposium: Design Representation, Cambridge. MA.

Gero, J. S., \& McNeill, T. (1998). An Approach to the Analysis of Design Protocols. Design Studies, 21(3), $21-61$.

Gibbs, J. R. W. (2005). Embodiment and Cognitive Science. Cambridge: Cambridge University Press.

Gibson, J. J. (1986). The Ecological Approach to Perception. Hillside, NJ: Lawrence Erlbaum Associates.

Goel, V. (1995). Sketches of Thought. Cambridge: MIT Press.

Goel, V., \& Pirolli, P. (1989). Motivating the notion of generic design within InformationProcessing Theory: The Design Problem Space. AI Magazine, 10 (1).

Goel, V., \& Pirolli, P. (1992). The Structure of Design Problem Spaces. Cognitive Science, $16,395-429$.

Golonka, S., \& Wilson, A. (2012). Gibson's ecological approach - a model for the benefits of a theory driven psychology. AVANT, III (2), 40-53.

Haupt, G. (2013). The cognitive dynamics of socio-technological thinking in the early phases of expert designers' design process. (PhD), University of Pretoria, Pretoria.

Ho, C.-H. (2001). Some Phenomena of Problem Decomposition Strategy for Design Thinking: Differences Between Novices and Experts. Design Studies, 22, 27-45.

Kearsley, G. (1998). Explorations in Learning \& Instruction: The Theory Into Practice Database: Gestalt Theory. Retrieved January 2004, 2004, from http://www.gwu.edu/ tip/wertheim.html

Kilgour, A. M. (2006). The Creative Process: The Effects of Domain Specific Knowledge and Creative Thinking Techniques on Creativity. University of Waikato, Waikato.

Kim, M. H., Kim, Y. S., Lee, H. S., \& Park, J. A. (2007). An Underlying Cognitive Aspect of Design Creativity: Limited Commitment Mode Control Strategy. Design Studies, 585604.

Kimbell, R. Stables, K., \& Green, R. (1996). Understanding Practice in Design and Technology. Buckingham: Open University Press.

Kirsh, D. (2009). Problem Solving and Situation Cognition. In P. Robbins \& M. Aydede (Eds.), The Cambridge Handbook of Situated Cognition. Cambridge: Cambridge University Press.

Lawson, B., \& Dorst, K. (2009). Design Expertise. Oxford: Architectural Press.

Liikkanen, L. A. (2009). Exploring Problem Decomposition in Conceptual Design Among Novice Designers. Design Studies, 30(1), 38 - 59.

Lovett, M. C., \& Anderson, J. R. (1996). History of Success and Current Context in Problem Solving. Cognitive Psychology, 31, 168-217.

Marsh, L., \& Drayson, Z. (2010). Extended Cognition and the Metaphysics of Mind. Cognitive Systems Research, 11, 367-377.

Miller, G. A., Galanter, E., \& Pribram, K. (1960). Plans and the Structure of Behaviour. New York: Holt, Rinehart \& Winston.

Natarajan, C. (2007). Culture and Technology Education. In M. De Vries, R. L. Custer, J. R. Dakers \& G. Martin (Eds.), Analyzing Best Practices in Technology Education. Rotterdam: Sense Publishers.

Newell, A., \& Simon, H. A. (1972). Human Problem Solving. Englewood Cliffs, N.J.: Prentice-Hall.

Oxman, R. (2002). The Thinking Eye: Visual Re-cognition in Design Emergence. Design Studies, 23(2), 135-164.

Petrina, S. (2007). Advanced Teaching Methods for the Technology Classroom. London: Information Science Publishing. 
Petrina, S., Feng, F., \& Kim, J. (2008). Researching Cognition and Technology: How We Learn Across the Lifespan. International Journal of Technology and Design Education, 18(4), 376-396.

Popovic, V. (2004). Expertise development in product design — strategic and domain-specific knowledge connections. Design Studies, 25, 527-545.

Reitman, W. R. (1964). Heuristic Decision Procedures, Open Constraints, and the Structure of Ill-defined Problems. In M. W. Shelly \& G. L. Bryan (Eds.), Human Judgements and Optimality. New York: Wiley.

Richardson, M. H., Shockley, K., Fajen, B. R., Riley, M. A., \& Turvey, M. T. (2008). Ecological Psychology: Six Principles for an Embodied-Embedded Approach to Behavior. In P. Calvo \& A. Gomila (Eds.), Handbook of cognitive science: an embodied approach. Amsterdam: Elsevier Science.

Savin-Baden, M. (2007). Challenging PBL Models and Perspectives. In E. de Graaf \& A. Kolmos (Eds.), Management of Change: Implementation of Problem-Based and Project-Based Learning in Engineering. Rotterdam: Sense Publishers.

Schön, D. A. (1987). Educating the Reflective Practitioner. San Francisco: Jossey-Bass.

Shani, I. (2012). Making it Mental: In Search for the Golden Mean of the Extended Cognition Controversy. Phenomenology and the Cognitive Sciences, September 26.

Simon, H. A. (1969). The Sciences of the Artificial (1st ed.). Cambridge MA: MIT Press.

Simon, H. A. (1996). The Sciences of the Artificial (3rd ed.). Cambridge, MA: MIT Press.

Simonton, D. K. (2003). Expertise, Competence, and Creative Ability: The Perplexing Complexities. In R. J. Sternberg \& E. L. Grigorenko (Eds.), The Psychology of Abilities, Competencies, and Expertise. Cambridge: Cambridge University Press.

Smith, L. B. (2005). Cognition As a Dynamic System: Principles from Embodiment. Developmental Review (25), 278-298.

Spiro, R. J., Feltovich, P. J., Jacobson, M. J., \& Coulson, R. L. (2013). Cognitive Flexibility, Constructivistm and Hypertext: Rondom access Instruction for Advanced Knowledge Acquisition in Ill-Structured Domains. In T. M. Duffy \& D. S. Jonassen (Eds.), Constructivism and the Technology of Instruction. Hillside, N.J.: Lawrence Earlbaum.

Stables, K. (1997). Critical Issues to Consider When Introducing Technology Education into the Curriculum of Young Learners. Journal of Technology Education. Retrieved 2, 8, from http://scholar.lib.vt.edu/ejournals/JTE/v8n2/stables.jte-v8n2.html.

Suwa, M., Purcell, T., \& Gero, J. (1998). Macroscopic Analysis of Design Processes Based on a Scheme for Coding Designers' Cognitive Actions. Design Studies, 19(4), 455483.

Suwa, M., \& Tversky, B. (1997). How Do Designers Shift Their Focus of Attention in Their Own Sketches? , 8. www-psych.stanford.edu/ bt/.../SuwaTversky.DRII.Word.do c1.pdf

Tashakkori, A., \& Teddlie, C. (2009). Foundations of Mixed Methods Research. Integrating Quantitative and Qualitative Approaches in the Social and Behavioural Sciences. London: Sage.

Terzidis, K. (2007). The etymology of design: pre-Socratic perspective. Design Issues. 23(4), 69-78.

Vincenti, W. G. (1990). What Engineers Know and How they Know it. Baltimore: John Hopkins Press.

Visser, W. (2004). Dynamic Aspects of Design Cognition: Elements for a Cognitive Model of Design (pp. 1-116). Rocquencourt: Institut National de Recherche en Informatique en Automatique 